\title{
Carleman Inequalities for Parabolic Equations in Sobolev Spaces of Negative Order and Exact Controllability for Semilinear Parabolic Equations
}

\author{
By \\ Oleg Yu. Imanuvilov* and Masahiro Yamamoto**
}

\begin{abstract}
We prove Carleman inequalities for a second order parabolic equation when the coefficients are not bounded and norms of right hand sides are taken in the Sobolev space $L^{2}\left(0, T ; W_{2}^{-\ell}(\Omega)\right), \ell \in[0,1]$. Our Carleman inequality yields the unique continuation for $L^{2}$-solutions. We further apply these inequalities to the global exact zero controllability of a semilinear parabolic equation whose semilinear term also contains derivatives of first order of solutions and is of sub-linear growth at the infinity.
\end{abstract}

Communicated by H. Okamoto. Received July 2, 2001. Revised April 3, 2002.

2000 Mathematics Subject Classification(s): 35K10, 35K55, 35K60, 93B05, 93C10, 93C20.

Key words and phrases: Carleman inequality, Sobolev space of negative order, unique continuation, exact controllability, duality, semilinear parabolic equation.

This research supported partly by the International Science Foundation and Government of Russia under grant number M 76300, GARC-KOSEF and KOSEF(K94073). The second named author is partly supported by grants 15340027 and 15654015 from the Japan Society for Promotion of Sciences and Sanwa Systems Development Co., Ltd. (Tokyo, Japan).

* Department of Mathematics, Iowa State University, 400 Carver Hall Ames IA 50011-2064 USA.

e-mail: vika@iastate.edu

** Department of Mathematical Sciences, The University of Tokyo, 3-8-1 Komaba Meguro Tokyo 153 Japan.

e-mail: myama@ms.u-tokyo.ac.jp 


\section{$\S 1 . \quad$ Introduction}

Since Carleman [3], there have been great concerns in Carleman inequalities. In particular, after the appearance of fundamental results by Hörmander [23], the theory is one of the most developing areas of linear partial differential equations. Among recent significant achievements, let us mention new unique continuation theorems for the hyperbolic operators in a spatial domain $\Omega$ and in a time interval $(0, T)$ (Hörmander [24], Robbiano [45], Ruiz [46], Tataru [52], [53], for example). Since [23], the theory has progressed in several directions, among which we mention the theory of Carleman inequalities in $L^{p}$-spaces with $p \neq 2$ (see Jerison and Kenig [31], Kim [32], [33], Sogge [49]) and the theory of Carleman inequalities with singular weight function. We can further refer to Jerison [30]. Note that these papers deal with either of the following "non-regular cases"

(1) Coefficients of low order terms belong to the space $L^{p}\left(0, T ; L^{q}(\Omega)\right)$ for some $p, q \in[1,+\infty]$ and right hand sides are taken in some $L^{p}$-space.

(2) Coefficients possess isolated singularities.

For Carleman inequalities for parabolic equations, see Isakov [28], [29], Kurata [34], Lavrent'ev, Romanov and Shishat.skiı̌ [36], Lin [37], Mizohata [42], Poon [44], Saut and Scheurer [48], Sogge [50], for example. In their works, coefficients of first order terms are assumed to be at least bounded, and coefficients of zero order term are assumed to be from the space $L^{p}\left(0, T ; L^{q}(\Omega)\right)$. Such a boundedness assumption makes the proof simple, but prevents us from applying the inequalities to solutions of semilinear parabolic equations which are less regular. In particular, Fabre [9], [10], Fabre and Lebeau [11] establish Carleman inequalities with norm of right hand sides in negative order Sobolev spaces for the Laplace and heat operators and functions with compact supports.

The first purpose of this paper is to establish Carleman inequalities for linear parabolic equations where the coefficients of terms of lower order are not regular and the right hand sides are in Sobolev spaces of negative orders. More precisely, we consider parabolic equations of the second order in a bounded cylindrical domain $Q=(0, T) \times \Omega$ with the zero Dirichlet boundary conditions on $\partial \Omega$, where the coefficients of the zeroth order term in the equations are in $L^{\infty}\left(0, T ; W_{r}^{-\kappa}(\Omega)\right)$ and the right hand sides are in the spaces $L^{2}\left(0, T ; W_{2}^{-\ell}(\Omega)\right)$ for some $r>1$ and $\ell \in[0,1]$. However for the principal part, we have to assume that the coefficients are Lipschitz continuous (see Corollary 2.1). As for less regular coefficients of second-order terms, we can refer to $\mathrm{Lu}$ [41], Wolff [55] in the case of elliptic equations. 
The technique in this paper is combinations of several methods. That is, the proof of the Carleman inequalities is based essentially on a duality argument, a theory of extremal problems, smoothing properties of parabolic and elliptic operators and $L^{2}$-Carleman inequalities proved by Chae, Imanuvilov and Kim [4], Imanuvilov [25].

Here we state other characteristics of our Carleman inequalities.

(1) Our Carleman inequalities hold over the whole domain $Q$, while classical Carleman inequalities are valid in subdomains bounded by level sets (e.g. Isakov [29], Lavrentiev, Romanov and Shishat.skiu [36]), or sufficiently small domains (e.g. Saut and Scheurer [48]).

(2) For our Carleman inequalities, the solutions have to satisfy the boundary condition on the whole boundary over the time interval. On the other hand, for classical Carleman inequalities, we can discuss solutions locally in the spatial domain $\Omega$ by introducing appropriate cut-off functions.

(3) Within solutions satisfying a boundary condition, our Carleman inequalities enable us to obtain unconditional global Lipschitz stability in a state estimation problem of determining a solution at a preceding time in terms of values of a solution in $(0, T) \times \omega$, where $\omega \subset \Omega$ is an arbitrary subdomain. On the other hand, classical Carleman inequalities cannot give such unconditional global Lipschitz stability estimates. Only Hölder stability can be proved (e.g. [29]).

Next we state the second purpose of this paper: applications of the Carleman inequalities. Firstly, with a suitable cut-off function, our Carleman inequalities imply the unique continuation theorem for parabolic operators within $L^{2}(Q)$-solutions. That is, if any $L^{2}(Q)$-solution of a parabolic equation with the zero right hand side vanishes in $(0, T) \times \mathcal{O} \subset Q$ where $\mathcal{O}$ is an arbitrary open subset of $\Omega$, then it identically vanishes in the whole domain $Q$ (Theorem 2.2 ). Simultaneously by our Carleman inequalities, we can prove the abovementioned unconditional and global Lipschitz stability for solutions at any intermediate time, provided that solutions satisfy the zero Dirichlet boundary condition.

Another important application of our Carleman inequalities is exact controllability of semilinear parabolic equations. In this paper we prove the exact zero controllability for semilinear parabolic equations of the second order where the nonlinear term depends on $(t, x) \in Q, y=y(t, x)$ and $\nabla y(t, x)$, and is of sub-linear growth at the infinity (see (3.7) and (3.8)). The methodology is same as in [20] and [25], but it relies on Carleman inequalities obtained in this paper. 
We can further refer to Guo and Littman [22] for the exact zero controllability for a parabolic equation whose semilinear term contains $\nabla y$ and satisfies analyticity condition.

In relation with the other controllability, in the beginning of 1990's, Fabre, Puel and Zuazua in [12], [13] have proved the global approximate controllability for second order semilinear parabolic equations with nonlinear term $f(t, x, y)$ of sub-linear growth in the variable $y$ at the infinity. Later the first named author of this paper proved the global exact controllability for the same equation ([25]). This result was improved by Fernández-Cara [15]. On the other hand, for the case of nonlinear term including $\nabla y$, the approximate controllability was established only recently by Fernández and Zuazua [14] and Zuazua [56]. For other important results on boundary controllability of evolution equations of fluid mechanics, see Coron [5]-[7], Coron and Fursikov [8], Fabre [9], [10], Fabre and Lebeau [11], Fursikov and Imanuvilov [16]-[21], Imanuvilov [26].

We conclude this section with a remark on further applications of Carleman inequalities to inverse problems. As is seen in Isakov [29, Chapter 8] for example, Carleman inequalities are useful for proving the uniqueness and stability in inverse problems of determining spatially varying coefficients in partial differential equations by overdetermining data on lateral boundary. In particular, thanks to the above-mentioned global character of our Carleman inequalities, we can prove Lipschitz stability which is global in the whole domain for the inverse problems. In Imanuvilov and Yamamoto [27], we establish such stability within $L^{2}$-coefficients for inverse parabolic problems, on the basis of the Carleman inequalities in usual $L^{2}$-spaces in [20], [25]. The Carleman inequalities proved in this paper, enable us to extend the results in [27] to inverse problems of determining less regular coefficients (not in $L^{2}(\Omega)$ ) and in a forthcoming paper, we will give details.

\section{$\S 2 . \quad$ Carleman Inequalities}

Let $(t, x) \in Q \equiv(0, T) \times \Omega, \Sigma \equiv(0, T) \times \partial \Omega$, where $\Omega \subset \mathbb{R}^{n}$ is a connected bounded domain whose boundary $\partial \Omega$ is sufficiently smooth, $\nu(x)$ is the external unit normal to $\partial \Omega, T \in(0,+\infty)$ is an arbitrary moment of time, $D^{\beta}=D^{\beta_{0}} D^{\beta^{\prime}}=\frac{\partial^{\beta_{0}}}{\partial t^{\beta_{0}}} \frac{\partial^{\beta_{1}}}{\partial x_{1}^{\beta_{1}}} \ldots \frac{\partial^{\beta_{n}}}{\partial x_{n}^{\beta_{n}}}, \beta=\left(\beta_{0}, \beta^{\prime}\right)=\left(\beta_{0}, \beta_{1}, \ldots, \beta_{n}\right)$, $|\beta|=2 \beta_{0}+\beta_{1}+\cdots+\beta_{n}$. Let $\omega \subset \Omega$ be an arbitrarily fixed subdomain and let us set $Q_{\omega}=(0, T) \times \omega$.

Throughout this paper, $W_{p}^{\mu}(\Omega)=W^{\mu, p}(\Omega), \stackrel{\circ}{W_{p}^{\mu}}(\Omega)=W_{0}^{\mu, p}(\Omega), p \geq 1$, $\mu \geq 0$ denote usual Sobolev spaces (e.g., Adams [1], Triebel [54]), and we set $L^{2}(\Omega)=W_{2}^{0}(\Omega)$. For non-integer $\ell=k+\gamma, k \in \mathbb{N} \cup\{0\}, \gamma \in(0,1)$, we note 
that

$$
\|u\|_{W_{p}^{\ell}(\Omega)}^{p}=\|u\|_{W_{p}^{k}(\Omega)}^{p}+\int_{\Omega} \int_{\Omega} \sum_{|\beta|=k} \frac{\left|D^{\beta} u(y)-D^{\beta} u(x)\right|^{p}}{|y-x|^{n+\gamma p}} d x d y .
$$

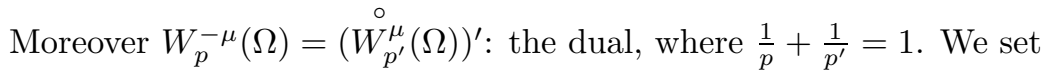

$$
\begin{aligned}
W^{1,2}(Q) & =\left\{y(t, x) \mid \frac{\partial y}{\partial t} \in L^{2}\left(0, T ; L^{2}(\Omega)\right), y \in L^{2}\left(0, T ; W_{2}^{2}(\Omega)\right)\right\}, \\
C^{1,2}(\bar{Q}) & =\left\{y=y(t, x) \mid y, \frac{\partial y}{\partial t}, \frac{\partial y}{\partial x_{i}}, \frac{\partial^{2} y}{\partial x_{i} \partial x_{j}} \in C(\bar{Q}), 1 \leq i, j \leq n\right\}
\end{aligned}
$$

and

$$
C^{0,1}(\bar{Q})=\left\{y=y(t, x) \mid y, \frac{\partial y}{\partial x_{i}} \in C(\bar{Q}), 1 \leq i \leq n\right\} .
$$

Henceforth $\mathcal{L}(X, Y)$ denotes the totality of bounded linear operators defined over a Banach space $X$ with values in another Banach space $Y$.

Let us consider the initial boundary value problem

$$
\begin{aligned}
& L y \equiv \frac{\partial y}{\partial t}-\sum_{i, j=1}^{n} \frac{\partial}{\partial x_{i}}\left(a_{i j}(t, x) \frac{\partial y}{\partial x_{j}}\right) \\
&+\sum_{i=1}^{n} \frac{\partial}{\partial x_{i}}\left(b_{i}(t, x) y\right)+c(t, x) y=g \text { in } Q, \\
&\left.y\right|_{\Sigma}=0, \quad y(0, \cdot)=y_{0} .
\end{aligned}
$$

Assume that

$$
\left\{\begin{array}{l}
a_{i j} \in W_{\infty}^{1}(Q), \quad a_{i j}=a_{j i}, \quad 1 \leq i, j \leq n, \\
b_{i} \in L^{\infty}\left(0, T ; L^{r}(\Omega)\right), \quad r>2 n, 1 \leq i \leq n, \\
c \in L^{\infty}\left(0, T ; W_{r_{1}}^{-\mu}(\Omega)\right), \quad 0 \leq \mu<\frac{1}{2}, \quad r_{1}>\max \left\{\frac{2 n}{3-2 \mu}, 1\right\},
\end{array}\right\}
$$

and the coefficients $a_{i j}$ satisfy the uniform ellipticity: There exists $\beta>0$ such that

$$
a(t, x, \zeta, \zeta) \equiv \sum_{i, j=1}^{n} a_{i j}(t, x) \zeta_{i} \zeta_{j} \geq \beta|\zeta|^{2}, \quad \zeta \in \mathbb{R}^{n}, \quad(t, x) \in Q .
$$

To formulate our Carleman inequality we need a special weight function.

Lemma 2.1 ([4], [25]). Let $\omega_{0} \subset \omega$ be an arbitrary fixed subdomain of $\Omega$ such that $\overline{\omega_{0}} \subset \omega$. Then there exists a function $\psi \in C^{2}(\bar{\Omega})$ such that

$$
\left\{\begin{array}{l}
\psi(x)>0 \quad \text { all } x \in \Omega,\left.\quad \psi\right|_{\partial \Omega}=0 \\
|\nabla \psi(x)|>0 \quad \text { for all } x \in \overline{\Omega \backslash \omega_{0} .}
\end{array}\right\}
$$


Now using the function $\psi$ constructed in Lemma 2.1, we introduce weight functions:

$$
\begin{aligned}
& \varphi(t, x)=e^{\lambda \psi(x)} /(t(T-t)), \quad \widehat{\varphi}(t)=1 /(t(T-t)), \\
& \alpha(t, x)=\left(e^{\lambda \psi(x)}-e^{2 \lambda\|\psi\|_{C(\bar{\Omega})}}\right) /(t(T-t)),
\end{aligned}
$$

where $\lambda>0$ is a parameter. Moreover we set

$$
\gamma=\sum_{i, j=1}^{n}\left\|a_{i j}\right\|_{W_{\infty}^{1}(Q)}+\sum_{i=1}^{n}\left\|b_{i}\right\|_{L^{\infty}\left(0, T ; L^{r}(\Omega)\right)}+\|c\|_{L^{\infty}\left(0, T ; W_{r_{1}}^{-\mu}(\Omega)\right)} .
$$

Denote by $L^{*}$ the operator formally adjoint to the operator $L$. Below we are dealing with weak $L^{2}$-solutions to the problem (2.1)-(2.2). Since under assumption (2.3), the function $c(t, x) y(t, x)$ is a distribution, we have to introduce the notion of weak solution to this problem using the method of transposition.

Definition 2.1. We say that $y \in L^{2}(Q)$ is a (weak) solution to the problem $(2.1)-(2.2)$ if for any $z \in L^{2}\left(0, T ; W_{2}^{1}(\Omega)\right)$ with $L^{*} z \in L^{2}(Q),\left.z\right|_{\partial \Omega}=0$ and $z(T, \cdot)=0$, the following equality holds true:

$$
\left(y, L^{*} z\right)_{L^{2}(Q)}=(g, z)_{L^{2}(Q)}+\left(y_{0}, z(0, \cdot)\right)_{L^{2}(\Omega)} .
$$

We are ready to state our main result, which establishes Carleman inequalities in Sobolev spaces of negative orders.

Theorem 2.1. $\quad$ Let (2.3)-(2.4) be fulfilled and the functions $\varphi, \alpha$ be defined by (2.6) and (2.7). Then there exists a number $\hat{\lambda}>0$ such that for an arbitrary $\lambda \geq \hat{\lambda}$, we can choose $s_{0}(\lambda)>0$ satisfying: there exists a constant $C_{1}>0$ such that for each $s \geq s_{0}(\lambda)$ the solution $y \in L^{2}(Q)$ to the problem (2.1) and (2.2) satisfies the following inequality:

$$
\begin{gathered}
\int_{Q}\left((s \varphi)^{1-2 \ell}|\nabla y|^{2}+(s \varphi)^{3-2 \ell} y^{2}\right) e^{2 s \alpha} d x d t \\
\leq C_{1}\left(\left\|g e^{s \alpha}\right\|_{L^{2}\left(0, T ; W_{2}^{-\ell}(\Omega)\right)}^{2}+\int_{Q_{\omega}}(s \varphi)^{3-2 \ell} y^{2} e^{2 s \alpha} d x d t\right), \\
\text { for all } s \geq s_{0}(\lambda), \quad \ell \in[0,1] .
\end{gathered}
$$

Here the constant $C_{1}$ is dependent continuously on $\gamma, \lambda$ and independent of $s$.

Moreover if $g(t, x)=g_{0}(t, x)+\sum_{i=1}^{n} \frac{\partial g_{i}(t, x)}{\partial x_{i}}$ with $g_{i} \in L^{2}(Q), 1 \leq i \leq n$, then the following estimate holds true for any $d \in \mathbb{R}$ :

$$
\int_{Q}\left((s \varphi)^{d-1}|\nabla y|^{2}+(s \varphi)^{d+1} y^{2}\right) e^{2 s \alpha} d x d t
$$




$$
\begin{gathered}
\leq C_{2}\left(\left\|g_{0}(s \varphi)^{\frac{d}{2}} e^{s \alpha}\right\|_{L^{2}\left(0, T ; W_{2}^{-1}(\Omega)\right)}^{2}\right. \\
\left.+\sum_{i=1}^{n}\left\|g_{i}(s \varphi)^{\frac{d}{2}} e^{s \alpha}\right\|_{L^{2}(Q)}^{2}+\int_{Q_{\omega}}(s \varphi)^{1+d} y^{2} e^{2 s \alpha} d x d t\right), \\
\text { for all } s \geq s_{0}(\lambda, d),
\end{gathered}
$$

where the constant $C_{2}>0$ is dependent continuously on $\gamma, \lambda, d$ and independent of $s$.

Corollary 2.1. $\quad$ The statement of Theorem 2.1 holds true if we assume that the coefficients $a_{i j}, 1 \leq i, j \leq n$ of the principal part are just Lipschitz continuous on $\bar{Q}$.

We postpone the proof of the corollary till the end of this section.

Carleman inequality (2.10) implies the following unique continuation result by a similar argument with using level sets of $\psi$ (e.g. [23], [29, Chapter 3]).

Theorem 2.2. Let the conditions in (2.3) hold for the coefficients $b_{i}$, $1 \leq i \leq n$, c. Moreover let the coefficients $a_{i j}$ with $a_{i j}=a_{j i}, 1 \leq i, j \leq n$ be Lipschitz continuous on $\bar{Q}$ and let (2.4) hold. Suppose that $y \in L^{2}(Q)$ is a solution to equation (2.1) with the right hand side $g \equiv 0$. If $y$ equals zero in $[0, T] \times \mathcal{O}$ where $\mathcal{O}$ is some open set in $\Omega$, then $y$ identically equals zero over the whole $Q$.

With more restrictive assumptions on regularity of coefficients of a parabolic operator, the Carleman inequality (2.9) with $\ell=0$ was proved in Chae, Imanuvilov and Kim [4], Fursikov and Imanuvilov [20], Imanuvilov [25], for example. Theorem 2.1 generalizes such Carleman inequalities.

The rest part of this section is devoted to the proof of Theorem 2.1. First we show

Lemma 2.2. Let $0<\mu<\frac{1}{2}$ and $r_{1}>\max \left\{\frac{2 n}{3-2 \mu}, 1\right\}, \frac{1}{r_{1}}+\frac{1}{r_{1}^{\prime}}=1$. Then there exist constants $0<\delta<\frac{1}{2}$ and $C>0$ such that

$$
\|z v\|_{W_{r_{1}^{\prime}}^{\mu}(\Omega)} \leq C\|v\|_{W_{2}^{1}(\Omega)}\|z\|_{W_{2}^{\frac{1}{2}-\delta}(\Omega)}
$$

for all $v \in \stackrel{\circ}{W_{2}^{1}}(\Omega)$ and $z \in W_{2}^{\frac{1}{2}-\delta}(\Omega)$.

The proof of the lemma is technical and so it is given in Appendix I. 
Lemma 2.3. Let $(2.3)-(2.4)$ be fulfilled, $b_{i} \in C^{0,1}(\bar{Q}), c \in L^{\infty}(Q)$ and the functions $\varphi, \alpha$ be defined by (2.6) and (2.7). Let $d \in \mathbb{R}$. Then there exists $\widehat{\lambda}>0$ such that for an arbitrary $\lambda \geq \hat{\lambda}$, we can choose $s_{0}=s_{0}(\lambda, d)>0$ satisfying: there exists a constant $C_{3}=C_{3}(\lambda, d)>0$ such that a solution $y \in L^{2}(Q)$ to problem (2.1) and (2.2) satisfies the following inequality:

$$
\begin{gathered}
\int_{Q}\left((s \varphi)^{1+d}|\nabla y|^{2}+(s \varphi)^{3+d} y^{2}\right) e^{2 s \alpha} d x d t \\
\leq C_{3}\left(\left\|(s \varphi)^{\frac{d}{2}} g e^{s \alpha}\right\|_{L^{2}(Q)}^{2}+\int_{Q_{\omega}}(s \varphi)^{3+d} y^{2} e^{2 s \alpha} d x d t\right) \\
\text { for all } s \geq s_{0}(\lambda, d),
\end{gathered}
$$

where the constant $C_{3}>0$ is independent of $s$.

Proof of Lemma 2.3. In the case of $d=0$, inequality (2.11) with $C^{1,2}$ coefficients $a_{i j}$ is shown, for example, in [4], [25]. For completeness, in Appendix II, we will give the proof in the case of $d=0$. Thus we have to prove (2.11) for $d \neq 0$. By taking a constant $C_{3}>0$ sufficiently large for $\lambda$ if necessary, it suffices to prove $(2.11)$ after the function $\varphi(t, x)$ is substituted by $\widehat{\varphi}(t)$ (see (2.6)). In fact, we can choose a constant $C_{3}^{\prime}>0$ such that

$$
\frac{1}{C_{3}^{\prime}} \widehat{\varphi}(t) \leq \varphi(t, x) \leq C_{3}^{\prime} \widehat{\varphi}(t), \quad(t, x) \in Q .
$$

Set $w(t, x)=y(t, x) \widehat{\varphi}(t)^{\frac{d}{2}}$. By $(2.1)$, the function $w$ satisfies

$$
L w=\frac{g}{(t(T-t))^{\frac{d}{2}}}-\frac{d}{2}\left(\frac{1}{t}-\frac{1}{T-t}\right) w=g \widehat{\varphi}^{\frac{d}{2}}-\frac{d}{2}(T-2 t) \widehat{\varphi} w
$$

and

$$
w_{\mid \Sigma}=0 .
$$

Applying to this equation the Carleman estimate (2.11) with $d=0$, we have

$$
\begin{gathered}
\int_{Q}\left(s \varphi|\nabla w|^{2}+(s \varphi)^{3} w^{2}\right) e^{2 s \alpha} d x d t \\
\leq C_{3}^{\prime \prime}\left(\left\|g \widehat{\varphi}^{\frac{d}{2}} e^{s \alpha}\right\|_{L^{2}(Q)}^{2}+\int_{Q} \widehat{\varphi}^{2} w^{2} e^{2 s \alpha} d x d t+\int_{Q_{\omega}}(s \varphi)^{3} w^{2} e^{2 s \alpha} d x d t\right) \\
\leq C_{3}\left(\left\|g \varphi^{\frac{d}{2}} e^{s \alpha}\right\|_{L^{2}(Q)}^{2}+\int_{Q} \varphi^{2} w^{2} e^{2 s \alpha} d x d t+\int_{Q_{\omega}}(s \varphi)^{3} w^{2} e^{2 s \alpha} d x d t\right), \\
\forall s \geq s_{0}(\lambda) .
\end{gathered}
$$


Then, increasing the parameter $s_{0}$ if necessary, we obtain

$$
\begin{gathered}
\int_{Q}\left(s \varphi|\nabla w|^{2}+(s \varphi)^{3} w^{2}\right) e^{2 s \alpha} d x d t \\
\leq C_{3}\left(\left\|g \varphi^{\frac{d}{2}} e^{s \alpha}\right\|_{L^{2}(Q)}^{2}+\int_{Q_{\omega}}(s \varphi)^{3} w^{2} e^{2 s \alpha} d x d t\right), \\
\text { for all } s \geq s_{0}(\lambda, d) .
\end{gathered}
$$

Consequently, the change of $w(t, x)=y(t, x) \hat{\varphi}(t)^{\frac{d}{2}}$ yields $(2.11)$.

Lemma 2.4. Let $y_{0} \in L^{2}(\Omega), g \in L^{2}\left(0, T ; W_{2}^{-1}(\Omega)\right)$ and conditions (2.3) be fulfilled for $b_{i}, 1 \leq i \leq n$ and $c$. Moreover let $a_{i j}=a_{j i}, 1 \leq i, j \leq n$ be Lipschitz continuous on $\bar{Q}$ and let (2.4) be satisfied. Then there exists a solution $y \in L^{2}\left(0, T ; \stackrel{\circ}{W_{2}^{1}}(\Omega)\right)$ to $(2.1)$ and $(2.2)$ which is unique in $L^{2}(Q)$, and the estimate is true:

$$
\|y\|_{L^{2}\left(0, T ; W_{2}^{1}(\Omega)\right) \cap C\left([0, T] ; L^{2}(\Omega)\right)} \leq C\left(\|y(0, \cdot)\|_{L^{2}(\Omega)}+\|g\|_{L^{2}\left(0, T ; W_{2}^{-1}(\Omega)\right)}\right),
$$

where the constant $C>0$ depends continuously only on parameter $\gamma$.

This lemma can be proved by a usual energy method (see, e.g., [35]) and, for completeness, we will give the proof in Appendix III.

Throughout this section, $C_{k}>0$ and $C>0$ denote generic constants which are independent of parameters $s, \lambda$ and functions to be estimated.

Remark 2.1. To simplify the situation one can further assume that $y(t, x)$ equals zero in some neighbourhoods of $t=T$ and $t=0$.

In fact, let us suppose that for such functions, estimate (2.9) is proved. Set

$$
\tau_{\varepsilon}(t)=\left\{\begin{array}{l}
0, \quad t \in[0, \varepsilon] \cup[T-\varepsilon, T] \\
\frac{t-\varepsilon}{\varepsilon}, \quad t \in(\varepsilon, 2 \varepsilon] \\
1, \quad t \in(2 \varepsilon, T-2 \varepsilon] \\
\frac{T-\varepsilon-t}{\varepsilon}, \quad t \in(T-2 \varepsilon, T-\varepsilon] .
\end{array}\right.
$$

By (2.9), the function $\tau_{\varepsilon}(t) y(t, x)$ satisfies the inequality

$$
\int_{Q}\left((s \varphi)^{1-2 \ell}|\nabla y|^{2}+(s \varphi)^{3-2 \ell} y^{2}\right) \tau_{\varepsilon}^{2} e^{2 s \alpha} d x d t
$$




$$
\begin{aligned}
\leq & C_{1}\left(\left\|g \tau_{\varepsilon} e^{s \alpha}\right\|_{L^{2}\left(0, T ; W_{2}^{-\ell}(\Omega)\right)}^{2}+\int_{\varepsilon}^{2 \varepsilon} \int_{\Omega} \frac{1}{\varepsilon^{2}} y^{2} e^{2 s \alpha} d x d t\right. \\
& \left.+\int_{T-2 \varepsilon}^{T-\varepsilon} \int_{\Omega} \frac{1}{\varepsilon^{2}} y^{2} e^{2 s \alpha} d x d t+\int_{Q_{\omega}}(s \varphi)^{3-2 \ell} y^{2} \tau_{\varepsilon}^{2} e^{2 s \alpha} d x d t\right),
\end{aligned}
$$

for all $s \geq s_{0}(\lambda)$ and $\ell \in[0,1]$. We note that there exists a constant $C_{4}>0$ independent of $\varepsilon>0$ and $(t, x) \in Q$, such that

$$
\int_{\varepsilon}^{2 \varepsilon} \int_{\Omega} \frac{1}{\varepsilon^{2}} y^{2} e^{2 s \alpha} d x d t \leq C_{4} \int_{\varepsilon}^{2 \varepsilon} \int_{\Omega} \frac{1}{\varepsilon^{2}} y^{2} \exp \left(-2 s \frac{C_{4}}{\varepsilon}\right) d x d t
$$

because $\alpha(t, x) \leq-\frac{C_{4}}{\varepsilon}, \varepsilon<t<2 \varepsilon, x \in \Omega$ by (2.7). Moreover a similar estimate holds for the third integral at the right hand side. Therefore, passing to the limit in this inequality as $\varepsilon \rightarrow 0$ and keeping in mind that $y \in L^{2}(Q)$, we obtain $(2.9)$.

Henceforth we set

$$
J(z, u)=\frac{1}{2} \int_{Q}(s \varphi)^{2-d} z^{2} e^{-2 s \alpha} d x d t+\frac{1}{2} \int_{Q_{\omega}}(s \varphi)^{-1-d} u^{2} e^{-2 s \alpha} d x d t .
$$

Now let us consider the following extremal problem:

$$
\inf _{(z, u) \in \mathcal{U}} J(z, u)
$$

where $\mathcal{U}$ is the totality of $(z, u) \in W^{1,2}(Q) \times L^{2}(Q)$ satisfying

$$
L^{*} z=(s \varphi)^{1+d} y e^{2 s \alpha}+\chi_{\omega} u,\left.\quad z\right|_{\Sigma}=0,
$$

and

$$
z(T, \cdot)=z(0, \cdot)=0
$$

Here $\lambda>\widehat{\lambda}, s>s_{0}(\lambda, d)$, the parameters $\widehat{\lambda}, s_{0}(\lambda, d)$ are defined in the estimate (2.11) with $d$ substituted by $d-2$ and

$$
L^{*} y \equiv-\frac{\partial y}{\partial t}-\sum_{i, j=1}^{n} \frac{\partial}{\partial x_{i}}\left(a_{i j}(t, x) \frac{\partial y}{\partial x_{j}}\right)-\sum_{i=1}^{n} b_{i}(t, x) \frac{\partial y}{\partial x_{i}}+c(t, x) y
$$

is an operator formally adjoint to the operator $L$. Here and henceforth $\chi_{\omega}$ denotes the characteristic function of $\omega$. 
Proposition 2.1. Let $b_{i} \equiv c \equiv 0,1 \leq i \leq n$, and let conditions (2.3) and (2.4) hold true. Then there exists a unique solution $(\widehat{z}, \widehat{u}) \in W^{1,2}(Q) \times$ $L^{2}(Q)$ to the extremal problem (2.14)-(2.16) and

$$
\begin{gathered}
\int_{Q} \sum_{|\beta| \leq 1}(s \varphi)^{2-2|\beta|-d}\left|D^{\beta} \widehat{z}\right|^{2} e^{-2 s \alpha} d x d t \\
+\int_{0}^{T}(s \hat{\varphi})^{2-2 \ell-d}\left\|\widehat{z} e^{-s \alpha}\right\|_{W_{2}^{\ell}(\Omega)}^{2} d t \\
+\int_{Q_{\omega}} \frac{\widehat{u}^{2}}{(s \varphi)^{1+d}} e^{-2 s \alpha} d x d t \leq C_{5} \int_{Q}(s \varphi)^{1+d} y^{2} e^{2 s \alpha} d x d t \\
\forall s \geq s_{0}(\lambda, d), \ell \in[0,1]
\end{gathered}
$$

Proof of Proposition 2.1. Since $y(t, x) \equiv 0$ in neighbourhoods of $t=T$ and $t=0$, the existence of an admissible element for this problem was proved in [25]. Thus, by standard arguments (see Alekseev, Tikhomirov and Fomin [2], Lions [38], [39] for example), one can prove the existence of a unique solution $(\widehat{z}, \widehat{u}) \in W^{1,2}(Q) \times L^{2}(Q)$ to the problem $(2.14)-(2.16)$.

We set

$$
L^{0} y \equiv \frac{\partial y}{\partial t}-\sum_{i, j=1}^{n} \frac{\partial}{\partial x_{i}}\left(a_{i j}(t, x) \frac{\partial y}{\partial x_{j}}\right) \quad \text { in } Q .
$$

In other words, $L^{0}$ is the principal part of $L$ defined by (2.1). We apply the Lagrange principle (see [2]) to the problem (2.14) where the admissible set $\mathcal{U}$ of $(z, u)$ is defined by $(2.16)$ and

$$
\left(L^{0}\right)^{*} z=(s \varphi)^{1+d} y e^{2 s \alpha}+\chi_{\omega} u,\left.\quad z\right|_{\Sigma}=0 .
$$

Then we obtain the optimality system for this problem:

$$
\begin{aligned}
\left(L^{0}\right)^{*} \widehat{z} & =(s \varphi)^{1+d} y e^{2 s \alpha}+\chi_{\omega} \widehat{u},\left.\quad \widehat{z}\right|_{\Sigma}=0, \quad \widehat{z}(T, \cdot)=\widehat{z}(0, \cdot)=0 \\
L^{0} p & =-(s \varphi)^{2-d} \widehat{z} e^{-2 s \alpha},\left.\quad p\right|_{\Sigma}=0,\left.\quad p\right|_{Q_{\omega}}=\frac{\widehat{u}}{(s \varphi)^{1+d}} e^{-2 s \alpha}
\end{aligned}
$$

Applying to (2.19) Carleman estimate (2.11) with $d$ substituted by $d-2$, we have

$$
\begin{aligned}
& \int_{Q}(s \varphi)^{1+d} p^{2} e^{2 s \alpha} d x d t \leq C_{6}\left(\int_{Q}(s \varphi)^{2-d} \widehat{z}^{2} e^{-2 s \alpha} d x d t\right. \\
& \left.\quad+\int_{Q_{\omega}} \frac{1}{(s \varphi)^{1+d}} \widehat{u}^{2} e^{-2 s \alpha} d x d t\right)=2 C_{6} J(\widehat{z}, \widehat{u}) \quad \text { for all } s \geq s_{0}(\lambda, d)
\end{aligned}
$$


Then taking scalar products of (2.18) with $p$ in $L^{2}(Q)$, integrating by parts and applying (2.19), we obtain

$$
\begin{aligned}
0 & =\int_{Q}\left(\left(L^{0}\right)^{*} \widehat{z}-(s \varphi)^{1+d} y e^{2 s \alpha}-\chi_{\omega} \widehat{u}\right) p d x d t \\
& =\int_{Q} L^{0} p \widehat{z} d x d t-\int_{Q_{\omega}} \frac{\widehat{u}^{2}}{(s \varphi)^{1+d}} e^{-2 s \alpha} d x d t-\int_{Q}(s \varphi)^{1+d} y p e^{2 s \alpha} d x d t \\
& =-2 J(\widehat{z}, \widehat{u})-\int_{Q}(s \varphi)^{1+d} y p e^{2 s \alpha} d x d t
\end{aligned}
$$

Hence, by the Cauchy-Bunyakovskii inequality,

$$
\begin{aligned}
J(\widehat{z}, \widehat{u}) & =\frac{1}{2} \int_{Q}(s \varphi)^{1+d} y p e^{2 s \alpha} d x d t \\
& \leq C_{7}\left(\int_{Q}(s \varphi)^{1+d} y^{2} e^{2 s \alpha} d x d t\right)^{\frac{1}{2}}\left(\int_{Q}(s \varphi)^{1+d} p^{2} e^{2 s \alpha} d x d t\right)^{\frac{1}{2}} .
\end{aligned}
$$

Substitution of (2.20) into this inequality yields

$$
\begin{aligned}
J(\widehat{z}, \widehat{u}) & =\frac{1}{2} \int_{Q}(s \varphi)^{2-d} \widehat{z}^{2} e^{-2 s \alpha} d x d t+\frac{1}{2} \int_{Q_{\omega}}(s \varphi)^{-1-d} \widehat{u}^{2} e^{-2 s \alpha} d x d t \\
& \leq C_{8} \int_{Q}(s \varphi)^{1+d} y^{2} e^{2 s \alpha} d x d t \quad \text { for all } s \geq s_{0}(\lambda, d) .
\end{aligned}
$$

Multiplying (2.18) by $(s \varphi)^{-d} e^{-2 s \alpha} \widehat{z}$ and integrating by parts, we obtain

$$
\begin{aligned}
\int_{Q}|\nabla \widehat{z}|^{2}(s \varphi)^{-d} e^{-2 s \alpha} d x d t & \\
\leq & C_{9}\left(\int_{Q}(s \varphi)^{2-d}|\widehat{z}|^{2} e^{-2 s \alpha} d x d t+\int_{Q}(s \varphi)^{1+d} y^{2} e^{2 s \alpha} d x d t\right. \\
& \left.\quad+\int_{Q} \frac{\widehat{u}^{2}}{(s \varphi)^{1+d}} e^{-2 s \alpha} d x d t\right) \\
\leq & C_{10} \int_{Q}(s \varphi)^{1+d} y^{2} e^{2 s \alpha} d x d t \quad \text { for all } s \geq s_{0}(\lambda, d) .
\end{aligned}
$$

In fact, applying integration by parts in (2.18), we have

$$
\begin{array}{r}
-\sum_{i, j=1}^{n} \int_{Q} \frac{\partial}{\partial x_{i}}\left(a_{i j} \frac{\partial \widehat{z}}{\partial x_{j}}\right)(s \varphi)^{-d} \widehat{z} e^{-2 s \alpha} d x d t \\
=\sum_{i, j=1}^{n} \int_{Q} a_{i j} \frac{\partial \widehat{z}}{\partial x_{i}} \frac{\partial \widehat{z}}{\partial x_{j}}(s \varphi)^{-d} e^{-2 s \alpha} d x d t
\end{array}
$$




$$
-\sum_{i, j=1}^{n} \int_{Q} a_{i j} \frac{\partial \widehat{z}}{\partial x_{i}} \widehat{z}(s \varphi)^{-d}\left(d \varphi^{-1} \varphi_{x_{j}}+2 s \alpha_{x_{j}}\right) e^{-2 s \alpha} d x d t
$$

and

$$
\begin{aligned}
-\int_{Q} \frac{\partial \widehat{z}}{\partial t} & (s \varphi)^{-d} \widehat{z} e^{-2 s \alpha} d x d t \\
& =\int_{Q} \widehat{z} \frac{\partial \widehat{z}}{\partial t}(s \varphi)^{-d} e^{-2 s \alpha} d x d t+\int_{Q} \widehat{z}^{2} \frac{\partial}{\partial t}\left((s \varphi)^{-d} e^{-2 s \alpha}\right) d x d t
\end{aligned}
$$

namely,

$$
-\int_{Q} \frac{\partial \widehat{z}}{\partial t}(s \varphi)^{-d} \widehat{z} e^{-2 s \alpha} d x d t=-\frac{1}{2} \int_{Q} \widehat{z}^{2}(s \varphi)^{-d}\left(d \varphi^{-1} \varphi_{t}+2 s \alpha_{t}\right) e^{-2 s \alpha} d x d t .
$$

Therefore the first equation in (2.18) implies

$$
\begin{aligned}
\int_{Q}\left(L^{0}\right)^{*} \widehat{z}(s \varphi)^{-d} \widehat{z} e^{-2 s \alpha} d x d t=-\frac{1}{2} \int_{Q} \widehat{z}^{2}(s \varphi)^{-d}\left(d \varphi^{-1} \varphi_{t}+2 s \alpha_{t}\right) e^{-2 s \alpha} d x d t \\
\quad+\sum_{i, j=1}^{n} \int_{Q} a_{i j} \frac{\partial \widehat{z}}{\partial x_{i}} \frac{\partial \widehat{z}}{\partial x_{j}}(s \varphi)^{-d} e^{-2 s \alpha} d x d t \\
\quad-\sum_{i, j=1}^{n} \int_{Q} a_{i j} \frac{\partial \widehat{z}}{\partial x_{i}} \widehat{z}(s \varphi)^{-d}\left(d \varphi^{-1} \varphi_{x_{j}}+2 s \alpha_{x_{j}}\right) e^{-2 s \alpha} d x d t \\
=\int_{Q} s \varphi y \widehat{z} d x d t+\int_{Q_{\omega}}(s \varphi)^{-d \widehat{u} \widehat{z} e^{-2 s \alpha} d x d t .}
\end{aligned}
$$

Hence

$$
\begin{aligned}
\sum_{i, j=1}^{n} \int_{Q} a_{i j} \frac{\partial \widehat{z}}{\partial x_{i}} \frac{\partial \widehat{z}}{\partial x_{j}}(s \varphi)^{-d} e^{-2 s \alpha} d x d t \\
=\int_{Q} s \varphi y \widehat{z} d x d t+\int_{Q_{\omega}}(s \varphi)^{-d} \widehat{u} \widehat{z} e^{-2 s \alpha} d x d t \\
\quad+\frac{1}{2} \int_{Q} \widehat{z}^{2}(s \varphi)^{-d}\left(d \varphi^{-1} \varphi_{t}+2 s \alpha_{t}\right) e^{-2 s \alpha} d x d t \\
\quad+\sum_{i, j=1}^{n} \int_{Q} a_{i j} \frac{\partial \widehat{z}}{\partial x_{i}} \widehat{z}(s \varphi)^{-d}\left(d \varphi^{-1} \varphi_{x_{j}}+2 s \alpha_{x_{j}}\right) e^{-2 s \alpha} d x d t
\end{aligned}
$$

On the other hand, we can see

$$
\left\{\begin{array}{l}
0<C_{11}^{-1} \leq \varphi \\
\left|\varphi_{t}\right| \leq C_{12} \varphi^{2}, \quad\left|\varphi_{x_{j}}\right| \leq C_{12} \varphi \\
\left|\alpha_{t}\right| \leq C_{12} \varphi^{2}, \quad\left|\alpha_{x_{j}}\right| \leq C_{12} \varphi \quad \text { in } Q
\end{array}\right\} .
$$


Therefore by the uniform ellipticity (2.4) and (2.23), for a small constant $\varepsilon>0$, we have

$$
\begin{aligned}
C_{13} \int_{Q}|\nabla \widehat{z}|^{2}(s \varphi)^{-d} e^{-2 s \alpha} d x d t \\
\leq \int_{Q}\left|(s \varphi)^{\frac{1+d}{2}} y e^{s \alpha}\right|\left|(s \varphi)^{\frac{2-d}{2}} \widehat{z} e^{-s \alpha}\right| d x d t \\
\quad+\int_{Q_{\omega}}\left|(s \varphi)^{-\frac{1+d}{2}} \widehat{u} e^{-s \alpha}\right|\left|(s \varphi)^{\frac{2-d}{2}} \widehat{z} e^{-s \alpha}\right| d x d t+\int_{Q}(s \varphi)^{2-d}|\widehat{z}|^{2} e^{-2 s \alpha} d x d t \\
\quad+\sum_{i, j=1}^{n} \int_{Q}\left|\varepsilon \frac{\partial \widehat{z}}{\partial x_{i}}(s \varphi)^{-\frac{d}{2}} e^{-s \alpha}\right|\left|\frac{1}{\varepsilon} \widehat{z}(s \varphi)^{-\frac{d-2}{2}} e^{-s \alpha}\right| d x d t \\
\leq\left(\int_{Q}(s \varphi)^{1+d} y^{2} e^{2 s \alpha} d x d t+\int_{Q}(s \varphi)^{2-d}|\widehat{z}|^{2} e^{-2 s \alpha} d x d t\right) \\
\quad+\left(\int_{Q_{\omega}} \frac{\widehat{u}^{2}}{(s \varphi)^{1+d}} e^{-2 s \alpha} d x d t+\int_{Q}(s \varphi)^{2-d}|\widehat{z}|^{2} e^{-2 s \alpha} d x d t\right) \\
\quad+\int_{Q}(s \varphi)^{2-d}|\widehat{z}|^{2} e^{-2 s \alpha} d x d t \\
\quad+\varepsilon^{2} \sum_{i, j=1}^{n} \int_{Q}\left|\frac{\partial \widehat{z}}{\partial x_{i}}\right|^{2}(s \varphi)^{-d} e^{-2 s \alpha} d x d t+\frac{1}{\varepsilon^{2}} \int_{Q}(s \varphi)^{2-d}|\widehat{z}|^{2} e^{-2 s \alpha} d x d t .
\end{aligned}
$$

Here we have used $|a b| \leq \frac{1}{2}|a|^{2}+\frac{1}{2}|b|^{2}$. Taking $\varepsilon>0$ sufficiently small, we obtain the first inequality in (2.22). The second inequality in (2.22) follows from (2.21). Consequently we have proved (2.17) for $|\beta| \leq 1$. Thus the proof of Proposition 2.1 is complete.

Proof of Theorem 2.1. Let $\widehat{z}$ and $\widehat{u}$ be the pair constructed in Proposition 2.1. Then by definition of weak solution, we have

$$
\begin{aligned}
0 & =\int_{Q} y\left(L^{*} \widehat{z}-g \widehat{z}\right) d x d t=\int_{Q}\left(y\left(L^{0}\right)^{*} \widehat{z}-\sum_{i=1}^{n} b_{i} y \frac{\partial \widehat{z}}{\partial x_{i}}+(c y-g) \widehat{z}\right) d x d t \\
& =\int_{Q}(s \varphi)^{1+d} y^{2} e^{2 s \alpha} d x d t+\int_{Q_{\omega}} \widehat{u} y d x d t-\int_{Q}\left(\sum_{i=1}^{n} b_{i} y \frac{\partial \widehat{z}}{\partial x_{i}}+(g-c y) \widehat{z}\right) d x d t .
\end{aligned}
$$

Hence

$$
\int_{Q}(s \varphi)^{1+d} y^{2} e^{2 s \alpha} d x d t=\int_{Q}\left(\sum_{i=1}^{n} b_{i} y \frac{\partial \widehat{z}}{\partial x_{i}}+(g-c y) \widehat{z}\right) d x d t-\int_{Q_{\omega}} \widehat{u} y d x d t .
$$


We can directly prove that

$$
\begin{aligned}
(2.25) \mid \int_{Q} & \left(\sum_{i=1}^{n} b_{i} y \frac{\partial \widehat{z}}{\partial x_{i}}-c y \widehat{z}\right) d x d t \mid \\
\leq & C(\varepsilon)\left(\sum_{i=1}^{n}\left\|b_{i} y(s \varphi)^{\frac{d}{2}} e^{s \alpha}\right\|_{L^{2}(Q)}^{2}+\left\|c y(s \varphi)^{\frac{d}{2}} e^{s \alpha}\right\|_{L^{2}\left(0, T ; W_{2}^{-1}(\Omega)\right)}^{2}\right) \\
& +\varepsilon\left(\left\|(\nabla \widehat{z}) e^{-s \alpha}(s \varphi)^{-\frac{d}{2}}\right\|_{L^{2}(Q)}^{2}+\left\|\widehat{z} e^{-s \alpha}(s \varphi)^{\frac{1-d}{2}}\right\|_{L^{2}(Q)}^{2}\right) .
\end{aligned}
$$

Let us estimate the first two terms at the right hand side of (2.25). Since $r>2 n$ and the Hölder inequality, we have

$$
\begin{aligned}
\sum_{i=1}^{n}\left\|b_{i} y(s \varphi)^{\frac{d}{2}} e^{s \alpha}\right\|_{L^{2}(Q)}^{2} & \\
& \leq C_{14}^{\prime} \int_{0}^{T} \sum_{i=1}^{n}\left\|b_{i}(t, \cdot)\right\|_{L^{r}(\Omega)}^{2}\left\|y(t, \cdot)(s \varphi)^{\frac{d}{2}} e^{s \alpha}\right\|_{L^{\frac{2 r}{r-2}}(\Omega)}^{2} d t \\
& \leq C_{14} \int_{0}^{T}\left\|y(t, \cdot)(s \varphi)^{\frac{d}{2}} e^{s \alpha}\right\|_{W_{2}^{\frac{1}{2}-\delta}(\Omega)}^{2} d t
\end{aligned}
$$

with some $0<\delta<\frac{r-2 n}{2 r}$.

By the Sobolev embedding theorem, we have

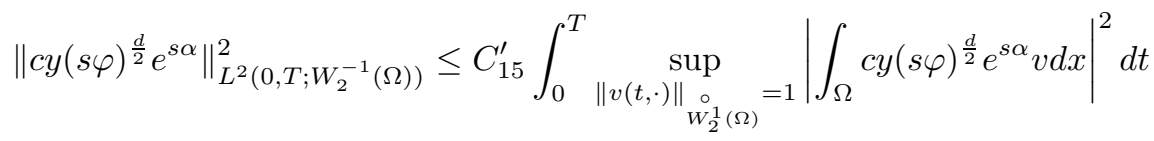

$$
\begin{aligned}
& \leq C_{15}^{\prime \prime} \int_{0}^{T}\|c\|_{L^{\infty}\left(0, T ; W_{r_{1}}^{-\mu}(\Omega)\right)}^{2} \sup _{\|v(t, \cdot)\|_{\substack{\circ \\
W_{2}^{1}(\Omega)}}}\left\|y(s \varphi)^{\frac{d}{2}} e^{s \alpha} v\right\|_{W_{r_{1}^{\prime}}^{\mu}(\Omega)}^{2} d t \\
& \leq C_{15} \int_{0}^{T} \sup _{\|v(t, \cdot)\|_{\substack{o \\
W_{2}^{1}(\Omega)}}}\left\|y(t, \cdot) v(t, \cdot)(s \varphi)^{\frac{d}{2}} e^{s \alpha}\right\|_{W_{r_{1}^{\prime}}^{\mu}(\Omega)}^{2} d t .
\end{aligned}
$$

Henceforth we take the 0 -extension of $y(s \varphi)^{\frac{d}{2}} e^{s \alpha}$ and $v$ outside $\Omega$. Then by Lemma 2.2, we obtain

$$
\left\|y(t, \cdot) v(t, \cdot)(s \varphi)^{\frac{d}{2}} e^{s \alpha}\right\|_{W_{r_{1}^{\prime}}^{\mu}(\Omega)}^{2} \leq C_{16}^{\prime}\|v(t, \cdot)\|_{W_{2}^{1}(\Omega)}\left\|y(s \varphi)^{\frac{d}{2}} e^{s \alpha}\right\|_{W_{2}^{\frac{1}{2}-\delta}(\Omega)}
$$

and so we see

$$
\left\|c y(s \varphi)^{\frac{d}{2}} e^{s \alpha}\right\|_{L^{2}\left(0, T ; W_{2}^{-1}(\Omega)\right)}^{2} \leq C_{16} \int_{0}^{T}\left\|y(s \varphi)^{\frac{d}{2}} e^{s \alpha}\right\|_{W_{2}^{\frac{1}{2}-\delta}(\Omega)}^{2} d t .
$$


From (2.25)-(2.27), we obtain

$$
\begin{aligned}
& \left|\int_{Q}\left(\sum_{i=1}^{n} b_{i} y \frac{\partial \widehat{z}}{\partial x_{i}}-c y \widehat{z}\right) d x d t\right| \leq C(\varepsilon) \int_{0}^{T}\left\|y(s \varphi)^{\frac{d}{2}} e^{s \alpha}\right\|_{W_{2}^{\frac{1}{2}-\delta}(\Omega)}^{2} d t \\
& +\varepsilon\left(\left\|(\nabla \widehat{z}) e^{-s \alpha}(s \varphi)^{-\frac{d}{2}}\right\|_{L^{2}(Q)}^{2}+\left\|\widehat{z} e^{-s \alpha}(s \varphi)^{\frac{1-d}{2}}\right\|_{L^{2}(Q)}^{2}\right) .
\end{aligned}
$$

Thanks to (2.17), noting that $|a b| \leq \frac{1}{2}|a|^{2}+\frac{1}{2}|b|^{2}$, we have

$$
\begin{aligned}
\left|\int_{Q_{\omega}} \widehat{u} y d x d t\right| & =\left|\int_{Q_{\omega}} \widehat{u}(s \varphi)^{-\frac{1+d}{2}} e^{-s \alpha} y(s \varphi)^{\frac{1+d}{2}} e^{s \alpha} d x d t\right| \\
& \leq \frac{1}{2} \int_{Q_{\omega}} \frac{\widehat{u}^{2}}{(s \varphi)^{1+d}} e^{-2 s \alpha} d x d t+\frac{1}{2} \int_{Q_{\omega}} y^{2}(s \varphi)^{1+d} e^{2 s \alpha} d x d t \\
& \leq \frac{C_{5}}{2} \int_{Q_{\omega}} y^{2}(s \varphi)^{1+d} e^{2 s \alpha} d x d t+\frac{1}{2} \int_{Q_{\omega}} y^{2}(s \varphi)^{1+d} e^{2 s \alpha} d x d t
\end{aligned}
$$

By (2.17), (2.28) and (2.29), we obtain from (2.24)

$$
\begin{aligned}
\int_{Q}(s \varphi)^{1+d} y^{2} e^{2 s \alpha} d x d t & \\
\leq & C_{17}\left(\left|\int_{Q} g \widehat{z} d x d t\right|+\int_{0}^{T}\left\|y(s \varphi)^{\frac{d}{2}} e^{s \alpha}\right\|_{W_{2}^{\frac{1}{2}-\delta}(\Omega)}^{2} d t\right. \\
& \left.+\int_{Q_{\omega}}(s \varphi)^{1+d} y^{2} e^{2 s \alpha} d x d t\right) \quad \text { for all } s \geq s_{0}(\lambda, d)
\end{aligned}
$$

Taking scalar products of $(2.1)$ with $(s \varphi)^{d-1} y e^{2 s \alpha}$ in $L^{2}(Q)$, we obtain

$$
\begin{aligned}
\int_{\Omega}\left(\int_{0}^{T} \frac{\partial y}{\partial t} y e^{2 s \alpha}(s \varphi)^{d-1} d t\right) d x & \\
& -\int_{0}^{T}\left(\int_{\Omega} \sum_{i, j=1}^{n} \frac{\partial}{\partial x_{i}}\left(a_{i j} \frac{\partial y}{\partial x_{j}}\right)(s \varphi)^{d-1} e^{2 s \alpha} y d x\right) d t \\
= & \int_{Q}\left(\sum_{i=1}^{n} b_{i} y \frac{\partial}{\partial x_{i}}\left((s \varphi)^{d-1} y e^{2 s \alpha}\right)-c y^{2}(s \varphi)^{d-1} e^{2 s \alpha}\right) d x d t \\
& +\int_{Q} g y e^{2 s \alpha}(s \varphi)^{d-1} d x d t
\end{aligned}
$$

By integration by parts, $\lim _{t \uparrow T} \alpha(t, x)=\lim _{t \downarrow 0} \alpha(t, x)=-\infty$ and $y_{\mid \Sigma}=0$, we have

$$
\int_{Q} y \frac{\partial y}{\partial t} e^{2 s \alpha}(s \varphi)^{d-1} d x d t=-\frac{1}{2} \int_{Q} y^{2} \frac{\partial}{\partial t}\left((s \varphi)^{d-1} e^{2 s \alpha}\right) d t d x
$$


and

$$
\begin{aligned}
-\int_{0}^{T} & \left(\int_{\Omega} \sum_{i, j=1}^{n} \frac{\partial}{\partial x_{i}}\left(a_{i j} \frac{\partial y}{\partial x_{j}}\right) y(s \varphi)^{d-1} e^{2 s \alpha} d x\right) d t \\
= & \int_{Q} \sum_{i, j=1}^{n} a_{i j} \frac{\partial y}{\partial x_{j}} \frac{\partial y}{\partial x_{i}}(s \varphi)^{d-1} e^{2 s \alpha} d x d t \\
& +\int_{Q} \sum_{i, j=1}^{n} a_{i j} \frac{\partial y}{\partial x_{j}} y \frac{\partial}{\partial x_{i}}\left((s \varphi)^{d-1} e^{2 s \alpha}\right) d x d t
\end{aligned}
$$

Hence

$$
\begin{aligned}
& \int_{Q} \sum_{i, j=1}^{n} a_{i j} \frac{\partial y}{\partial x_{j}} \frac{\partial y}{\partial x_{i}}(s \varphi)^{d-1} e^{2 s \alpha} d x d t=\frac{1}{2} \int_{Q} y^{2} \frac{\partial}{\partial t}\left((s \varphi)^{d-1} e^{2 s \alpha}\right) d x d t \\
- & \int_{Q} \sum_{i, j=1}^{n} a_{i j} \frac{\partial y}{\partial x_{j}} y \frac{\partial}{\partial x_{i}}\left((s \varphi)^{d-1} e^{2 s \alpha}\right) d x d t \\
+ & \int_{Q}\left(\sum_{i=1}^{n} b_{i} y \frac{\partial}{\partial x_{i}}\left((s \varphi)^{d-1} y e^{2 s \alpha}\right)-c y^{2}(s \varphi)^{d-1} e^{2 s \alpha}\right) d x d t \\
+ & \int_{Q} g y(s \varphi)^{d-1} e^{2 s \alpha} d x d t
\end{aligned}
$$

By (2.26) and (2.27), in terms of the Cauchy-Bunyakovkii inequality, we have

$$
\begin{aligned}
\mid \int_{Q} & \left\{\sum_{i=1}^{n} b_{i} y \frac{\partial}{\partial x_{i}}\left((s \varphi)^{d-1} y e^{2 s \alpha}\right)-c y^{2}(s \varphi)^{d-1} e^{2 s \alpha}\right\} d x d t \mid \\
\leq & C(\varepsilon)\left(\sum_{i=1}^{n}\left\|b_{i} y(s \varphi)^{\frac{d}{2}} e^{s \alpha}\right\|_{L^{2}(Q)}^{2}+\left\|c y(s \varphi)^{\frac{d}{2}} e^{s \alpha}\right\|_{L^{2}\left(0, T ; W_{2}^{-1}(\Omega)\right)}^{2}\right) \\
& +\varepsilon \int_{Q}(s \varphi)^{-d} e^{-2 s \alpha}\left|\frac{\partial}{\partial x_{i}}\left((s \varphi)^{d-1} y e^{2 s \alpha}\right)\right|^{2} d x d t \\
& +\varepsilon \sum_{i=1}^{n} \int_{Q}\left|\frac{\partial}{\partial x_{i}}\left(y e^{s \alpha}(s \varphi)^{\frac{d-2}{2}}\right)\right|^{2} d x d t \\
\leq & C(\varepsilon) \int_{0}^{T}\left\|y(s \varphi)^{\frac{d}{2}} e^{s \alpha}\right\|_{W_{2}^{\frac{1}{2}-\delta}(\Omega)}^{2} d t \\
& +\varepsilon \int_{Q}\left(|\nabla y|^{2}(s \varphi)^{d-1} e^{2 s \alpha}+(s \varphi)^{1+d} y^{2} e^{2 s \alpha}\right) d x d t .
\end{aligned}
$$


By (2.23), we have

$$
\left|\frac{\partial}{\partial t}\left((s \varphi)^{d-1} e^{2 s \alpha}\right)\right| \leq C_{18}(s \varphi)^{d+1} e^{2 s \alpha}
$$

and

$$
\left|\frac{\partial}{\partial x_{i}}\left((s \varphi)^{d-1} e^{2 s \alpha}\right)\right| \leq C_{18}(s \varphi)^{d} e^{2 s \alpha}, \quad 1 \leq i \leq n
$$

for large $s>0$. Therefore, in terms of (2.4), using (2.31)-(2.34), we obtain

$$
\begin{aligned}
\int_{Q} & |\nabla y|^{2}(s \varphi)^{d-1} e^{2 s \alpha} d x d t \leq C_{19} \int_{Q} y^{2}(s \varphi)^{d+1} e^{2 s \alpha} d x d t \\
& +C_{19} \int_{Q}\left((s \varphi)^{\frac{d-1}{2}}|\nabla y| e^{s \alpha}\right)\left((s \varphi)^{\frac{d+1}{2}} y e^{s \alpha}\right) d x d t \\
& +C_{19}\left|\int_{Q} g e^{s \alpha}(s \varphi)^{d-1} y e^{s \alpha} d x d t\right| \\
& +C(\varepsilon) \int_{0}^{T}\left\|y(s \varphi)^{\frac{d}{2}} e^{s \alpha}\right\|_{W_{2}^{\frac{1}{2}-\delta}(\Omega)}^{2} d t \\
& +\varepsilon \int_{Q}\left(|\nabla y|^{2}(s \varphi)^{d-1} e^{2 s \alpha}+(s \varphi)^{1+d} y^{2} e^{2 s \alpha}\right) d x d t
\end{aligned}
$$

In (2.35), we note that

$$
\begin{aligned}
& \left|\int_{Q} g e^{s \alpha}(s \varphi)^{d-1} y e^{s \alpha} d x d t\right| \\
& \leq C(\varepsilon)\left\|g(s \varphi)^{\frac{d}{2}} e^{s \alpha}\right\|_{L^{2}\left(0, T ; W_{2}^{-1}(\Omega)\right)}^{2}+\varepsilon\left\|\nabla\left((s \varphi)^{\frac{d}{2}-1} y e^{s \alpha}\right)\right\|_{L^{2}\left(0, T ; L^{2}(\Omega)\right)}^{2} \\
& \leq C(\varepsilon)\left\|g(s \varphi)^{\frac{d}{2}} e^{s \alpha}\right\|_{L^{2}\left(0, T ; W_{2}^{-1}(\Omega)\right)}^{2} \\
& \quad+\varepsilon \int_{Q}(s \varphi)^{d-1}|\nabla y|^{2} e^{2 s \alpha} d x d t+C \varepsilon \int_{Q}(s \varphi)^{d+1} y^{2} e^{2 s \alpha} d x d t .
\end{aligned}
$$

Consequently, combining (2.30) and (2.35) and taking a sufficiently small $\varepsilon>0$, in terms of the Cauchy-Bunyakovskii inequality, we obtain

$$
\begin{aligned}
& I(s) \equiv \int_{Q}\left(|\nabla y|^{2}(s \varphi)^{d-1}+(s \varphi)^{1+d} y^{2}\right) e^{2 s \alpha} d x d t \\
& \leq C_{20}\left(\left|\int_{Q} g \widehat{z} d x d t\right|+\left\|g(s \varphi)^{\frac{d}{2}} e^{s \alpha}\right\|_{L^{2}\left(0, T ; W_{2}^{-1}(\Omega)\right)}^{2}\right. \\
&\left.+\int_{0}^{T}\left\|y(s \varphi)^{\frac{d}{2}} e^{s \alpha}\right\|_{W_{2}^{\frac{1}{2}-\delta}(\Omega)}^{2} d t+\int_{Q_{\omega}}(s \varphi)^{1+d} y^{2} e^{2 s \alpha} d x d t\right) \\
& \text { for all } s \geq s_{0}(\lambda, d) .
\end{aligned}
$$


We can prove an interpolation inequality:

$$
s^{2 \delta}\left\|y(s \varphi)^{\frac{d}{2}} e^{s \alpha}\right\|_{L^{2}\left(0, T ; W_{2}^{\frac{1}{2}-\delta}(\Omega)\right)}^{2} \leq C_{21} I(s) \quad \text { whenever } \delta \in\left(0, \frac{1}{2}\right)
$$

so that we obtain from $(2.36)$

$$
\begin{aligned}
& I(s)=\int_{Q}\left(|\nabla y|^{2}(s \varphi)^{d-1}+(s \varphi)^{1+d} y^{2}\right) e^{2 s \alpha} d x d t \\
& \leq C_{22}\left(\left|\int_{Q} g \widehat{z} d x d t\right|+\left\|g(s \varphi)^{\frac{d}{2}} e^{s \alpha}\right\|_{L^{2}\left(0, T ; W_{2}^{-1}(\Omega)\right)}^{2}+\int_{Q_{\omega}}(s \varphi)^{1+d} y^{2} e^{2 s \alpha} d x d t\right) \\
& \text { for all } s \geq s_{0}(\lambda, d) .
\end{aligned}
$$

Let $g=g_{0}+\sum_{i=1}^{n} \frac{\partial}{\partial x_{i}} g_{i}$. Then $\int_{Q} g \widehat{z} d x d t=\int_{Q}\left(g_{0} \widehat{z}-\sum_{i=1}^{n} g_{i} \frac{\partial \widehat{z}}{\partial x_{i}}\right) d x d t$.

Consequently, by (2.17) and the Cauchy-Bunyakovskii inequality, for any $\varepsilon>0$, there exists a constant $C_{23}(\varepsilon)>0$ such that

$$
\begin{aligned}
& \left|\int_{Q} g \widehat{z} d x d t\right| \leq\left\|g_{0}(s \varphi)^{\frac{d}{2}} e^{s \alpha}\right\|_{L^{2}\left(0, T ; W_{2}^{-1}(\Omega)\right)}\left\|(s \varphi)^{-\frac{d}{2}} e^{-s \alpha} \widehat{z}\right\|_{L^{2}\left(0, T ; W_{2}^{1}(\Omega)\right)} \\
& \quad+\sum_{i=1}^{n}\left\|g_{i}(s \varphi)^{\frac{d}{2}} e^{s \alpha}\right\|_{L^{2}(Q)}\left\|\nabla \widehat{z}(s \varphi)^{-\frac{d}{2}} e^{-s \alpha}\right\|_{L^{2}(Q)} \\
& \leq C_{23}(\varepsilon)\left\|g_{0}(s \varphi)^{\frac{d}{2}} e^{s \alpha}\right\|_{L^{2}\left(0, T ; W_{2}^{-1}(\Omega)\right)}^{2} \\
& \quad+C_{23}(\varepsilon) \sum_{i=1}^{n}\left\|g_{i}(s \varphi)^{\frac{d}{2}} e^{s \alpha}\right\|_{L^{2}(Q)}^{2}+\varepsilon \int_{Q}(s \varphi)^{1+d} y^{2} e^{2 s \alpha} d x d t
\end{aligned}
$$

Thus (2.10) follows from (2.37) and (2.38).

Let $d=2-2 \ell$. We note that $\hat{\varphi}(t)$ is defined by (2.6). Then the duality, the Hölder inequality and the interpolation inequality (e.g. [1]), yield

$$
\begin{aligned}
& \left|\int_{Q} g e^{s \alpha}(s \varphi)^{1-2 \ell} y e^{s \alpha} d x d t\right| \\
& \quad \leq \frac{1}{\varepsilon}\left\|g e^{s \alpha}\right\|_{L^{2}\left(0, T ; W_{2}^{-\ell}(\Omega)\right)} \times \varepsilon\left\|(s \varphi)^{1-2 \ell} y e^{s \alpha}\right\|_{L^{2}\left(0, T ; W_{2}^{\ell}(\Omega)\right)}
\end{aligned}
$$

and

$$
\begin{aligned}
& \varepsilon\left\|(s \varphi)^{1-2 \ell} y e^{s \alpha}(t, \cdot)\right\|_{W_{2}^{\ell}(\Omega)} \\
& \quad \leq C_{24} \varepsilon\left\{(s \widehat{\varphi})^{\frac{\ell-2 \ell^{2}}{2}}\left\|y e^{s \alpha}(t, \cdot)\right\|_{W_{2}^{1}(\Omega)}^{\ell}\right\}\left\{(s \widehat{\varphi})^{1-2 \ell-\frac{\ell-2 \ell^{2}}{2}}\left\|y e^{s \alpha}(t, \cdot)\right\|_{L^{2}(\Omega)}^{1-\ell}\right\}
\end{aligned}
$$




$$
\begin{aligned}
& \leq C_{24} \varepsilon(s \widehat{\varphi})^{\frac{1-2 \ell}{2}}\left\|y e^{s \alpha}(t, \cdot)\right\|_{W_{2}^{1}(\Omega)}+C_{24} \varepsilon(s \widehat{\varphi})^{\frac{2 \ell^{2}-5 \ell+2}{2(1-\ell)}}\left\|y e^{s \alpha}(t, \cdot)\right\|_{L^{2}(\Omega)} \\
& \leq C_{25} \varepsilon\left\|(s \varphi)^{\frac{1-2 \ell}{2}} y e^{s \alpha}(t, \cdot)\right\|_{W_{2}^{1}(\Omega)}+C_{25} \varepsilon\left\|(s \varphi)^{\frac{3-2 \ell}{2}} y e^{s \alpha}(t, \cdot)\right\|_{L^{2}(\Omega)} .
\end{aligned}
$$

Here and henceforth, we have also used $C_{3}^{\prime} \widehat{\varphi}(t) \leq \varphi(t, x) \leq C_{3}^{\prime \prime} \widehat{\varphi}(t)$ for $(t, x) \in \bar{Q}$ and $\frac{2 \ell^{2}-5 \ell+2}{2(1-\ell)} \leq \frac{3-2 \ell}{2}$.

Therefore we have

$$
\begin{aligned}
& \left|\int_{Q} g e^{s \alpha}(s \varphi)^{1-2 \ell} y e^{s \alpha} d x d t\right| \\
& \quad \leq \frac{1}{\varepsilon^{2}}\left\|g e^{s \alpha}\right\|_{L^{2}\left(0, T ; W_{2}^{-\ell}(\Omega)\right)}+C_{26} \varepsilon^{2} \int_{Q}\left((s \varphi)^{1-2 \ell}|\nabla y|^{2}+(s \varphi)^{3-2 \ell} y^{2}\right) e^{2 s \alpha} d x d t .
\end{aligned}
$$

Then, similarly to (2.37), we apply (2.35) and (2.39) where we choose $\varepsilon>0$ sufficiently small, so that we obtain

$$
\begin{aligned}
\int_{Q}\left((s \varphi)^{1-2 \ell}|\nabla y|^{2}+(s \varphi)^{3-2 \ell} y^{2}\right) e^{2 s \alpha} d x d t \\
\leq C_{27}\left(\int_{0}^{T}\left\|\hat{z} e^{-s \alpha}\right\|_{W_{2}^{\ell}(\Omega)}^{2} d t\right)^{\frac{1}{2}}\left(\int_{0}^{T}\left\|g e^{s \alpha}\right\|_{W_{2}^{-\ell}(\Omega)}^{2} d t\right)^{\frac{1}{2}} \\
\quad+C_{27} \int_{Q_{\omega}}(s \varphi)^{3-2 \ell} y^{2} e^{2 s \alpha} d x d t \\
\leq C_{27}\left\|g e^{s \alpha}\right\|_{L^{2}\left(0, T ; W_{2}^{-\ell}(\Omega)\right)}^{2}+C_{27} \int_{Q_{\omega}}(s \varphi)^{3-2 \ell} y^{2} e^{2 s \alpha} d x d t
\end{aligned}
$$

This inequality implies (2.9). The proof of theorem is complete.

Proof of Corollary 2.1. We will approximate $a_{i j}$ by $W_{\infty}^{1}$-functions with the aid of the mollifiers (e.g. Adams [1]). Let $\kappa \in C^{\infty}\left(\mathbb{R}^{n+1}\right), \int_{\mathbb{R}^{n+1}} \kappa(t, x) d t d x$ $=1, \kappa(t, x) \geq 0$ for all $(t, x) \in \mathbb{R}^{n+1}$ and $\operatorname{supp} \kappa \subset\{(t, x)||(t, x) \mid \leq 1\}$. Set $a_{i j}^{\varepsilon}(t, x)=\frac{1}{\varepsilon^{n+1}} \int_{\mathbb{R}^{n+1}} \kappa\left(\frac{t-t^{\prime}}{\varepsilon}, \frac{x-x^{\prime}}{\varepsilon}\right) a_{i j}\left(t^{\prime}, x^{\prime}\right) d t^{\prime} d x^{\prime}$.

Then, since $a_{i j}$ are Lipschitz continuous on $\bar{Q}$, we can see that

$$
a_{i j}^{\varepsilon} \rightarrow a_{i j} \quad \text { in } C(\bar{Q}), \quad\left\|a_{i j}^{\varepsilon}\right\|_{W_{\infty}^{1}(Q)} \leq C, \quad 1 \leq i, j \leq n, \varepsilon>0 .
$$

Here $C>0$ is independent of $\varepsilon>0$. Therefore for $\left\{a_{i j}^{\varepsilon}\right\}_{1 \leq i, j \leq n}$ with any $\varepsilon>0$, the constant $\gamma$ in (2.8) is bounded and (2.4) is true with the same $\beta>0$. Let $L^{\varepsilon}$ be the linear parabolic operator obtained from $L$ after change of the coefficients $a_{i j}$ by $a_{i j}^{\varepsilon}$. Let us consider the boundary value problem

$$
L^{\varepsilon} y_{\varepsilon}=g \quad \text { in } Q,\left.\quad y_{\varepsilon}\right|_{\Sigma}=0, y_{\varepsilon}(0, \cdot)=0
$$


By (2.40) and Lemma 2.4, we can prove that

$$
y_{\varepsilon} \rightarrow y \quad \text { in } L^{2}\left(0, T ; W_{2}^{1}(\Omega)\right) \cap C\left([0, T] ; L^{2}(\Omega)\right),
$$

as $\varepsilon \longrightarrow 0$, where $y(t, x)$ is a solution to $(2.1)$ and (2.2) with $y_{0}=0$. Moreover by Theorem 2.1 for a solution to (2.41), inequalities (2.9) and (2.10) hold true with the constants $C_{1}$ and $C_{2}$ independent of $\varepsilon$. Passing to the limit in these inequalities and keeping in mind (2.42), we complete the proof of corollary.

\section{$\S 3 . \quad$ Exact Controllability of Semilinear Parabolic Equations}

Henceforth $a_{i j}, b_{i}, 1 \leq i, j \leq n$ and $c$ are assumed to satisfy (2.3). We consider the semilinear parabolic equation

$$
\begin{aligned}
G(y)= & \frac{\partial y}{\partial t}-\sum_{i, j=1}^{n} \frac{\partial}{\partial x_{i}}\left(a_{i j}(t, x) \frac{\partial y}{\partial x_{j}}\right)+\sum_{i=1}^{n} b_{i}(t, x) \frac{\partial y}{\partial x_{i}}(t, x) \\
& +c(t, x) y+f(t, x, \nabla y, y)=u+g \quad \text { in } Q \text { with } u \in \mathcal{U}(\omega)
\end{aligned}
$$

and

$$
\left.y\right|_{\Sigma}=0, \quad y(0, x)=v_{0}(x),
$$

where $v_{0}$ and $g$ are given, and $u(t, x)$ is a locally distributed control in the space

$$
\mathcal{U}(\omega)=\left\{u(t, x) \in L^{2}(Q) \mid \operatorname{supp} u \subset \overline{Q_{\omega}}\right\} .
$$

By the exact controllability, we mean a problem of finding a control $u \in \mathcal{U}(\omega)$ such that

$$
y(T, x)=v_{1}(x), \quad x \in \Omega,
$$

where $v_{1}(x)$ is a given function.

In this paper we also consider the exact boundary controllability, by which we mean a problem of finding a boundary control $u(t, x)$ such that

$$
\begin{aligned}
& G(y)=g \text { in } Q, \quad y(0, x)=v_{0}(x), \quad y(T, x)=v_{1}(x), \\
& \left.y\right|_{] 0, T\left[\times \Gamma_{0}\right.}=u,\left.\quad y\right|_{] 0, T\left[\times\left(\partial \Omega \backslash \Gamma_{0}\right)\right.}=0,
\end{aligned}
$$

where $\Gamma_{0} \subset \partial \Omega$ is an arbitrary fixed subboundary, and $v_{0}, v_{1}, g$ are given functions.

For a semilnear term $f$, let us assume that

$$
f\left(t, x, \zeta^{\prime}, \zeta_{0}\right) \in C^{1}\left(\bar{Q} \times \mathbb{R}^{n+1}\right), \quad f(t, x, 0,0)=0, \quad \forall(t, x) \in Q,
$$


and

$$
\left|\frac{\partial f\left(t, x, \zeta^{\prime}, \zeta_{0}\right)}{\partial \zeta_{i}}\right| \leq K, \quad \forall(t, x) \in Q, \quad \forall \zeta \equiv\left(\zeta^{\prime}, \zeta_{0}\right)=\left(\zeta_{1}, \ldots, \zeta_{n}, \zeta_{0}\right) \in \mathbb{R}^{n+1}
$$

for $0 \leq i \leq n$. Set

$$
\eta(t, x)=\left(-e^{\lambda \psi(x)}+e^{2 \lambda\|\psi\|_{C(\bar{\Omega})}}\right) /((T-t) \ell(t)),
$$

where

$$
\ell \in C^{\infty}[0, T], \quad \ell(t)>0, \ell(t) \geq t, \quad \forall t \in[0, T] \text { and } \ell(t)=t, \quad \forall t \in\left[\frac{T}{2}, T\right] .
$$

We set

$$
\begin{aligned}
L_{0} y= & \frac{\partial y}{\partial t}-\sum_{i, j=1}^{n} \frac{\partial}{\partial x_{i}}\left(a_{i j}(t, x) \frac{\partial y}{\partial x_{j}}\right) \\
& +\sum_{i=1}^{n} b_{i}(t, x) \frac{\partial y}{\partial x_{i}}(t, x)+c(t, x) y, \quad(t, x) \in Q .
\end{aligned}
$$

Henceforth we define a weighted $L^{2}$-space with a weight function $\kappa(t, x)>$ 0 for almost all $(t, x) \in Q$ :

$$
L^{2}(Q, \kappa)=\left\{\left.y\left|\int_{Q}\right| y(t, x)\right|^{2} \kappa(t, x) d x d t<\infty\right\}
$$

with the norm

$$
\|y\|_{L^{2}(Q, \kappa)}=\left(\int_{Q}|y(t, x)|^{2} \kappa(t, x) d x d t\right)^{\frac{1}{2}} .
$$

Now, in order to formulate our results, we introduce the function spaces

$$
\begin{aligned}
& X_{s}^{\lambda}(Q)=L^{2}\left(Q,(T-t) e^{2 s \eta}\right), \\
& Z_{s}^{\lambda}(Q)=\left\{y|y|_{\Sigma}=0, y, \nabla y \in L^{2}\left(Q, e^{2 s \eta}\right), \quad L_{0} y \in X_{s}^{\lambda}(Q)\right\}
\end{aligned}
$$

with the norm

$$
\|y\|_{Z_{s}^{\lambda}(Q)}^{2}=\left\|L_{0} y\right\|_{X_{s}^{\lambda}(Q)}^{2}+\|y\|_{L^{2}\left(Q, e^{2 s \eta}\right)}^{2}+\|\nabla y\|_{\left(L^{2}\left(Q, e^{2 s \eta}\right)\right)^{n}}^{2},
$$

and

$(3.15) \quad Y(Q)=\left\{y(t, x)\left|L_{0} y \in L^{2}\left(0, T ; L^{2}(\Omega)\right), y\right|_{\Sigma}=0, y(0, \cdot) \in W_{2}^{1}(\Omega)\right\}$ 
with the norm

$$
\|y\|_{Y(Q)}^{2}=\left\|L_{0} y\right\|_{L^{2}\left(0, T ; L^{2}(\Omega)\right)}^{2}+\|y(0, \cdot)\|_{W_{2}^{1}(\Omega)}^{2} .
$$

We state our main result which is the global exact zero controllability for semilinear parabolic equation (3.1).

Theorem 3.1. Let $v_{0} \in \stackrel{\circ}{W_{2}^{1}}(\Omega), v_{1} \equiv 0$, and let conditions (2.3)-(2.4), (3.7) and (3.8) be fulfilled. Then there exists $\widehat{\lambda}>0$ such that for $\lambda \geq \widehat{\lambda}$ there exists a constant $s_{0}(\lambda)$ such that for $g \in X_{s}^{\lambda}(Q)$, with $\lambda \geq \widehat{\lambda}, s \geq s_{0}(\lambda)$ there exists a solution pair $(y, u) \in Y(Q) \times \mathcal{U}(\omega)$ to (3.1), (3.2) and (3.4).

First we prove the existence of solution for a controllability problem in the case of linear parabolic equation.

$$
L_{0} y=g+u, \quad u \in \mathcal{U}(\omega),\left.y\right|_{\Sigma}=0, y(0, x)=v_{0}, y(T, x)=0 .
$$

We have

Lemma 3.1. Let $\lambda \geq \widehat{\lambda}$ and $v_{0} \in \stackrel{\circ}{W_{2}^{1}}(\Omega), v_{1} \equiv 0$, and let conditions (2.3)-(2.4) be fulfilled. Then there exists a constant $s_{0}(\lambda)>0$ such that if $g \in X_{s}^{\lambda}(Q)$ with $s \geq s_{0}(\lambda)$, then the problem (3.17) has a solution $(y, u) \in$ $\left(Y(Q) \cap Z_{s}^{\lambda}(Q)\right) \times\left(\mathcal{U}(\omega) \cap X_{s}^{\lambda}(Q)\right)$ which satisfies the following estimate:

$$
\|(y, u)\|_{\left(Y(Q) \cap Z_{s}^{\lambda}(Q)\right) \times\left(\mathcal{U}(\omega) \cap X_{s}^{\lambda}(Q)\right)} \leq C(\lambda, s, \gamma)\left(\left\|v_{0}\right\|_{W_{2}^{1}(\Omega)}+\|g\|_{X_{s}^{\lambda}(Q)}\right) .
$$

Proof. We recall that the parameters $\widehat{\lambda}$ and $s_{0}(\lambda)$ were defined in Theorem 2.1. For $k \in \mathbb{N}$, let us consider the extremal problem

$$
\begin{aligned}
\mathcal{J}_{k}(y, u)= & \frac{1}{2} \int_{Q} \rho_{k}\left(|\nabla y|^{2}+y^{2}\right) d x d t \\
& +\frac{1}{2} \int_{Q}(T-t) e^{2 s \eta(t, x)} m_{k} u^{2} d x d t \rightarrow \inf , \\
L_{0} y & =g+u \quad \text { in } Q,\left.y\right|_{\Sigma}=0, \quad y(0, x)=v_{0}, \quad y(T, x)=0,
\end{aligned}
$$

where

$$
\rho_{k}(t, x)=\exp \left(\frac{2 s \eta(t, x)(T-t)}{T-t+1 / k}\right), \quad m_{k}(x)=\left\{\begin{array}{l}
1, x \in \bar{\omega} \\
k, x \in \Omega \backslash \bar{\omega}
\end{array}\right.
$$


and the parameters $s \geq s_{0}(\lambda), \lambda \geq \hat{\lambda}$ are fixed.

It is easy to prove (see Lions [38], [39]) that the problem (3.19)-(3.20) has a unique solution, which we denote by $\left(\widehat{y}_{k}, \widehat{u}_{k}\right) \in Y(Q) \times L^{2}(Q)$.

Applying the Lagrange principle to the problem (3.19)-(3.20) (see [2], [38]), we obtain

$$
\begin{aligned}
& L_{0} \widehat{y}_{k}=g+\widehat{u}_{k} \quad \text { in } Q,\left.\quad \widehat{y}_{k}\right|_{\Sigma}=0, \widehat{y}_{k}(T, \cdot) \equiv 0, \widehat{y}_{k}(0, \cdot)=v_{0}, \\
& L_{0}^{*} p_{k}=\nabla \cdot\left(\rho_{k} \nabla \widehat{y}_{k}\right)-\rho_{k} \widehat{y}_{k} \quad \text { in } Q \\
& \left.p_{k}\right|_{\Sigma}=0, p_{k}-(T-t) e^{2 s \eta(t, x)} m_{k} \widehat{u}_{k}=0 \quad \text { in } Q .
\end{aligned}
$$

Henceforth $C=C(\lambda, s)>0$ denotes a generic constant which is dependent on $\lambda$ and $s$, but independent of $k$.

For the proof of Lemma 3.1, we will prove

\section{Lemma 3.2.}

$$
\begin{aligned}
& \int_{Q} e^{-2 s \eta} \frac{\left|p_{k}\right|^{2}}{T-t} d x d t+\int_{\Omega}\left|p_{k}(0, x)\right|^{2} d x \\
& \quad \leq C(\lambda, s)\left(\int_{Q} \rho_{k}^{2} e^{-2 s \eta}\left(\left|\nabla \widehat{y}_{k}\right|^{2}+\left|\widehat{y}_{k}\right|^{2}\right) d x d t+\int_{Q_{\omega}} e^{-2 s \eta} \frac{p_{k}^{2}}{T-t} d x d t\right) .
\end{aligned}
$$

Proof of Lemma 3.2. First applying to (3.23) estimate (2.10) with $d=0$, we have

$\int_{Q} s \varphi\left|p_{k}\right|^{2} e^{2 s \alpha} d x d t \leq C \int_{Q} \rho_{k}^{2} e^{2 s \alpha}\left(\left|\nabla \widehat{y}_{k}\right|^{2}+\left|\widehat{y}_{k}\right|^{2}\right) d x d t+C \int_{Q_{\omega}} s \varphi\left|p_{k}\right|^{2} e^{2 s \alpha} d x d t$.

Hence

$$
\begin{aligned}
& \int_{Q} \frac{1}{t(T-t)}\left|p_{k}\right|^{2} e^{2 s \alpha} d x d t \\
& \quad \leq C \int_{Q} \rho_{k}^{2} e^{2 s \alpha}\left(\left|\nabla \widehat{y}_{k}\right|^{2}+\left|\widehat{y}_{k}\right|^{2}\right) d x d t+C \int_{Q_{\omega}} \frac{1}{t(T-t)}\left|p_{k}\right|^{2} e^{2 s \alpha} d x d t
\end{aligned}
$$

By definition (3.9), we have

$$
-\eta(t, x)=\alpha(t, x), \quad \frac{T}{2} \leq t \leq T
$$

and

$$
-\eta(t, x) \geq \alpha(t, x), \quad(t, x) \in Q .
$$


Moreover we have

$$
\frac{1}{t} e^{2 s \alpha} \leq C(s) e^{-2 s \eta}, \quad 0<t<T
$$

where $C(s)>0$ depends on $s>0$. In fact, this is equivalent to

$$
\mu_{s}(x, t) \equiv \frac{1}{t} \exp \left(2 s\left(\frac{1}{t(T-t)}-\frac{1}{(T-t) \ell(t)}\right)\left(e^{\lambda \psi(t, x)}-e^{\left.2 \lambda\|\psi\|_{C(\bar{\Omega})}\right)}\right) \leq C(s)\right.
$$

for $0 \leq t \leq T$ and $x \in \bar{\Omega}$. For this, it is sufficient to verify $\lim _{t \rightarrow 0} \mu_{s}(x, t)<\infty$ for $s>0$ and $x \in \bar{\Omega}$. By $\ell(t) \geq t$ for $0 \leq t \leq T$ and $\ell(0)>0$ from (3.10), we can directly see that

$$
\lim _{t \rightarrow 0} \mu_{s}(x, t)=0
$$

for $s>0$ and $x \in \bar{\Omega}$.

Therefore

$$
\begin{aligned}
& \int_{\frac{T}{2}}^{T} \int_{\Omega} \frac{1}{t(T-t)}\left|p_{k}\right|^{2} e^{-2 s \eta} d x d t \\
& \quad \leq C \int_{Q} \rho_{k}^{2} e^{-2 s \eta}\left(\left|\nabla \widehat{y}_{k}\right|^{2}+\left|\widehat{y}_{k}\right|^{2}\right) d x d t+C \int_{Q_{\omega}} \frac{1}{T-t}\left|p_{k}\right|^{2} e^{-2 s \eta} d x d t
\end{aligned}
$$

Let $\chi=\chi(t) \in C^{\infty}[0, T]$ be a function such that $1 \geqq \chi(t) \geq 0$ for $t \in[0, T]$, $\chi(t)=1$ for $t \in\left[0, \frac{T}{2}\right], \chi(t)=0$ for $t \geq \frac{3 T}{4}$. Multiplying (3.23) by $p_{k} \chi$ and taking scalar products in $L^{2}(\Omega)$ and integrating by parts, we obtain

$$
\begin{aligned}
- & \frac{1}{2} \frac{d}{d t} \int_{\Omega} \chi p_{k}^{2} d x+\int_{\Omega} \sum_{i, j=1}^{n} a_{i j} \frac{\partial p_{k}}{\partial x_{i}} \frac{\partial p_{k}}{\partial x_{j}} \chi(t) d x=-\frac{1}{2} \int_{\Omega} \frac{d \chi}{d t} p_{k}^{2} d x \\
& -\int_{\Omega}\left(\sum_{i=1}^{n} b_{i} p_{k} \chi \frac{\partial p_{k}}{\partial x_{i}}+c p_{k}^{2} \chi\right) d x-\int_{\Omega}\left(\rho_{k} \chi \nabla \widehat{y}_{k} \cdot \nabla p_{k}+\rho_{k} \widehat{y}_{k} p_{k} \chi(t)\right) d x .
\end{aligned}
$$

Here, similarly to (2.26), we use the interpolation inequality (e.g., [1]) to obtain

$$
\begin{aligned}
\left|\int_{\Omega} b_{i} p_{k} \chi \frac{\partial p_{k}}{\partial x_{i}} d x\right| & \leq\left\|b_{i} p_{k} \sqrt{\chi}\right\|_{L^{2}(\Omega)}\left\|\frac{\partial p_{k}}{\partial x_{i}} \sqrt{\chi}\right\|_{L^{2}(\Omega)} \\
& \leq C\left\|p_{k} \sqrt{\chi}\right\|_{W_{2}^{\frac{1}{2}-\delta}(\Omega)}\left\|\frac{\partial p_{k}}{\partial x_{i}} \sqrt{\chi}\right\|_{L^{2}(\Omega)} \\
& \leq C\left(\varepsilon\left\|p_{k} \sqrt{\chi}\right\|_{W_{2}^{1}(\Omega)}+C(\varepsilon)\left\|p_{k} \sqrt{\chi}\right\|_{L^{2}(\Omega)}\right) \times\left\|p_{k} \sqrt{\chi}\right\|_{W_{2}^{1}(\Omega)}
\end{aligned}
$$




$$
\leq C \varepsilon\left\|p_{k} \sqrt{\chi}\right\|_{W_{2}^{1}(\Omega)}^{2}+C(\varepsilon)\left\|p_{k} \sqrt{\chi}\right\|_{L^{2}(\Omega)}^{2} .
$$

Moreover, by (2.3), Lemma 2.2 and the interpolation inequality, we see that

$$
\begin{aligned}
\left|\int_{\Omega} c \chi p_{k}^{2} d x\right| & \leq\|c\|_{W_{r_{1}}^{-\mu}(\Omega)}\left\|\sqrt{\chi} p_{k} \sqrt{\chi} p_{k}\right\|_{W_{r_{1}^{\prime}}^{\mu}(\Omega)} \\
& \leq C\left\|\sqrt{\chi} p_{k}\right\|_{W_{2}^{1}(\Omega)}\left\|\sqrt{\chi} p_{k}\right\|_{W_{2}^{\frac{1}{2}-\delta}(\Omega)} \\
& \leq C\left\|\sqrt{\chi} p_{k}\right\|_{W_{2}^{1}(\Omega)}\left(\varepsilon\left\|\sqrt{\chi} p_{k}\right\|_{W_{2}^{1}(\Omega)}+C(\varepsilon)\left\|\sqrt{\chi} p_{k}\right\|_{L^{2}(\Omega)}\right) \\
& \leq \varepsilon\left\|\sqrt{\chi} p_{k}\right\|_{W_{2}^{1}(\Omega)}^{2}+C(\varepsilon)\left\|\sqrt{\chi} p_{k}\right\|_{L^{2}(\Omega)}^{2} .
\end{aligned}
$$

Noting (2.4) and

$$
2\left|\rho_{k} \chi \nabla \widehat{y}_{k} \cdot \nabla p_{k}\right| \leq 2\left|\rho_{k} \sqrt{\chi} \nabla \widehat{y}_{k} \cdot \nabla p_{k}\right| \leq \varepsilon \chi\left|\nabla p_{k}\right|^{2}+\frac{1}{\varepsilon} \rho_{k}^{2}\left|\nabla \widehat{y}_{k}\right|^{2},
$$

and taking sufficiently small $\varepsilon>0$, we apply (2.4), (3.27) and (3.28) to (3.26), so that we obtain

$$
\begin{aligned}
-\frac{1}{2} \frac{d}{d t} \int_{\Omega} \chi p_{k}^{2} d x & +\int_{\Omega}\left|\nabla p_{k}\right|^{2} \chi(t) d x \\
& \leq C \int_{\Omega}\left\{\left(\left|\frac{d \chi}{d t}\right|+\chi\right) p_{k}^{2}+\rho_{k}^{2}\left(\left|\nabla \widehat{y}_{k}\right|^{2}+\left|\widehat{y}_{k}\right|^{2}\right)\right\} d x,
\end{aligned}
$$

that is,

$$
\frac{d f}{d t}(t) \leq C f(t)+C \int_{\Omega}\left|\frac{d \chi}{d t}\right| p_{k}^{2} d x+C \int_{\Omega} \rho_{k}^{2}\left(\left|\nabla \widehat{y}_{k}\right|^{2}+\left|\widehat{y}_{k}\right|^{2}\right) d x, \quad 0 \leq t \leq T,
$$

where $f(t)=\int_{\Omega} \chi p_{k}^{2} d x$. Let us consider inequality (3.29) on the time interval $\left[0, \frac{3 T}{4}\right]$. Note that $\chi\left(\frac{3 T}{4}\right) p_{k}\left(\frac{3 T}{4}, \cdot\right)=0$. Applying the Gronwall inequality to (3.29) and taking into account that $\frac{d \chi}{d t}(t)=0,0 \leq t \leq \frac{T}{2}$, we obtain

$$
\begin{aligned}
f(t) & \leq C \int_{0}^{\frac{3 T}{4}} \int_{\Omega}\left|\frac{d \chi}{d t}\right| p_{k}^{2} d x d t+C \int_{0}^{\frac{3 T}{4}} \int_{\Omega} \rho_{k}^{2}\left(\left|\nabla \widehat{y}_{k}\right|^{2}+\left|\widehat{y}_{k}\right|^{2}\right) d x d t \\
& \leq C \int_{\frac{T}{2}}^{\frac{3 T}{4}} \int_{\Omega} p_{k}^{2} d x+C \int_{0}^{\frac{3 T}{4}} \int_{\Omega} \rho_{k}^{2}\left(\left|\nabla \widehat{y}_{k}\right|^{2}+\left|\widehat{y}_{k}\right|^{2}\right) d x d t
\end{aligned}
$$

that is,

$$
\int_{0}^{\frac{T}{2}} \int_{\Omega} p_{k}^{2} d x d t+\left\|p_{k}(0, \cdot)\right\|_{L^{2}(\Omega)}^{2}
$$




$$
\begin{aligned}
& \leq C \int_{\frac{T}{2}}^{\frac{3 T}{4}} \int_{\Omega} p_{k}^{2} d x d t+C \int_{0}^{\frac{3 T}{4}} \int_{\Omega} \rho_{k}^{2}\left(\left|\nabla \widehat{y}_{k}\right|^{2}+\left|\widehat{y}_{k}\right|^{2}\right) d x d t \\
& \leq C \int_{\frac{T}{2}}^{\frac{3 T}{4}} \int_{\Omega} p_{k}^{2} d x d t+C \int_{0}^{\frac{3 T}{4}} \int_{\Omega} e^{-2 s \eta} \rho_{k}^{2}\left(\left|\nabla \widehat{y}_{k}\right|^{2}+\left|\widehat{y}_{k}\right|^{2}\right) d x d t .
\end{aligned}
$$

Now inequalities (3.25) and (3.30) imply

$$
\begin{aligned}
& \int_{0}^{\frac{T}{2}} \int_{\Omega} p_{k}^{2} d x d t+\left\|p_{k}(0, \cdot)\right\|_{L^{2}(\Omega)}^{2} \\
& \quad \leq C(s)\left(\int_{Q} e^{-2 s \eta} \rho_{k}^{2}\left(\left|\nabla \widehat{y}_{k}\right|^{2}+\left|\widehat{y}_{k}\right|^{2}\right) d x d t+\int_{Q_{\omega}} \frac{1}{T-t} p_{k}^{2} e^{-2 s \eta} d x d t\right) .
\end{aligned}
$$

Hence

$$
\begin{aligned}
& \int_{0}^{\frac{T}{2}} \int_{\Omega} \frac{1}{T-t} p_{k}^{2} e^{-2 s \eta} d x d t+\left\|p_{k}(0, \cdot)\right\|_{L^{2}(\Omega)}^{2} \\
& \quad \leq C(\lambda, s)\left(\int_{Q} e^{-2 s \eta} \rho_{k}^{2}\left(\left|\nabla \widehat{y}_{k}\right|^{2}+\left|\widehat{y}_{k}\right|^{2}\right) d x d t+\int_{Q_{\omega}} \frac{1}{T-t} p_{k}^{2} e^{-2 s \eta} d x d t\right) .
\end{aligned}
$$

On the other hand, it follows from (3.25) that

$$
\begin{aligned}
& \int_{\frac{T}{2}}^{T} \int_{\Omega} \frac{1}{T-t} p_{k}^{2} e^{-2 s \eta} d x d t \\
& \quad \leq C(\lambda, s)\left(\int_{Q} e^{-2 s \eta} \rho_{k}^{2}\left(\left|\nabla \widehat{y}_{k}\right|^{2}+\left|\widehat{y}_{k}\right|^{2}\right) d x d t+\int_{Q_{\omega}} \frac{1}{T-t} p_{k}^{2} e^{-2 s \eta} d x d t\right) .
\end{aligned}
$$

This inequality and (3.31) complete the proof of Lemma 3.2.

We observe that $\left|\rho_{k}(t, x) e^{-2 s \eta(t, x)}\right| \leq 1,(t, x) \in Q$. Thus, by (3.24) and (3.23), we have

$$
\begin{aligned}
& \int_{\Omega}\left|p_{k}(0, x)\right|^{2} d x+\int_{Q} \frac{\left|p_{k}\right|^{2}}{T-t} e^{-2 s \eta} d x d t \\
& \quad \leq C(\lambda, s)\left(\int_{Q} \rho_{k}\left(\left|\nabla \widehat{y}_{k}\right|^{2}+y_{k}^{2}\right) d x d t+\int_{Q_{\omega}} e^{2 s \eta}(T-t) \widehat{u}_{k}^{2} d x d t\right) .
\end{aligned}
$$

Multiplying (3.23) by $\widehat{y}_{k}$, taking scalar products in $L^{2}(Q)$ and integrating by parts with respect to $t$ and $x$, we have

$$
0=\left(L_{0}^{*} p_{k}-\nabla \cdot\left(\rho_{k} \nabla \widehat{y}_{k}\right)+\rho_{k} \widehat{y}_{k}, \widehat{y}_{k}\right)_{L^{2}(Q)}=\int_{Q} \rho_{k}\left(\left|\nabla \widehat{y}_{k}\right|^{2}+\widehat{y}_{k}^{2}\right) d x d t
$$




$$
\begin{aligned}
& +\left(p_{k}, L_{0} \widehat{y}_{k}\right)_{L^{2}(Q)}+\left(p_{k}(0, \cdot), \widehat{y}_{k}(0, \cdot)\right)_{L^{2}(\Omega)}=\int_{Q} \rho_{k}\left(\left|\nabla \widehat{y}_{k}\right|^{2}+\widehat{y}_{k}^{2}\right) d x d t \\
& +\int_{Q}(T-t) e^{2 s \eta(t, x)} m_{k} \widehat{u}_{k}^{2} d x d t+\int_{Q} g p_{k} d x d t+\left(p_{k}(0, \cdot), v_{0}\right)_{L^{2}(\Omega)} .
\end{aligned}
$$

Hence

$$
\begin{aligned}
\mathcal{J}_{k}\left(\widehat{y}_{k}, \widehat{u}_{k}\right) & =\frac{1}{2} \int_{Q}\left(\rho_{k}\left(\left|\nabla \widehat{y}_{k}\right|^{2}+\widehat{y}_{k}^{2}\right)+(T-t) e^{2 s \eta(t, x)} m_{k} \widehat{u}_{k}^{2}\right) d x d t \\
& =\frac{1}{2}\left(-\int_{Q} g p_{k} d x d t-\left(p_{k}(0, \cdot), v_{0}\right)_{L^{2}(Q)}\right) .
\end{aligned}
$$

By (3.32) and (3.33) we obtain

$$
\mathcal{J}_{k}\left(\widehat{y}_{k}, \widehat{u}_{k}\right) \leq C\left(\|g\|_{X_{s}^{\lambda}(Q)}+\left\|v_{0}\right\|_{L^{2}(\Omega)}\right) \sqrt{\mathcal{J}_{k}\left(\widehat{y}_{k}, \widehat{u}_{k}\right)}
$$

It follows that

$$
\mathcal{J}_{k}\left(\widehat{y}_{k}, \widehat{u}_{k}\right) \leq C^{2}\left(\|g\|_{X_{s}^{\lambda}(Q)}+\left\|v_{0}\right\|_{L^{2}(\Omega)}\right)^{2} .
$$

By virtue of (3.34), we have a subsequence $\left\{\left(\widehat{y}_{k}, \widehat{u}_{k}\right)\right\}_{k=1}^{\infty}$ such that

$$
\begin{gathered}
\left(\widehat{y}_{k}, \widehat{u}_{k}\right) \rightarrow(y, u) \quad \text { weakly in } Y(Q) \times L^{2}(Q), \\
\widehat{u}_{k} \rightarrow 0 \quad \text { in } L^{2}((0, T) \times(\Omega \backslash \omega)), \\
e^{s \eta} \widehat{u}_{k} \rightarrow e^{s \eta} u \quad \text { weakly in } L^{2}(Q \omega), \\
\left(\sqrt{\rho_{k}} \frac{\partial \widehat{y}_{k}}{\partial x_{i}}, \sqrt{\rho_{k}} \widehat{y}_{k}\right) \rightarrow\left(e^{s \eta} \frac{\partial y}{\partial x_{i}}, e^{s \eta} y\right) \quad \text { weakly in } L^{2}((0, T-\varepsilon) \times \Omega) \quad \forall \varepsilon>0 .
\end{gathered}
$$

Using (3.35), we pass to the limit in (3.22) and obtain that pair $(y, u)$ is a solution to the problem (3.17). Estimate (3.18) follows from (3.34), (3.35) and Fatou's theorem.

Lemma 3.3. The imbedding $Y(Q) \subset L^{2}\left(0, T ; W_{2}^{1}(\Omega)\right)$ is compact.

Proof of Lemma 3.3. Let

$$
\left\|y_{k}\right\|_{Y(Q)} \equiv\left(\left\|L_{0} y_{k}\right\|_{L^{2}\left(0, T ; L^{2}(\Omega)\right)}^{2}+\|y(0, \cdot)\|_{W_{2}^{1}(\Omega)}^{2}\right)^{\frac{1}{2}} \leq C,
$$

for $k \in \mathbb{N}$. Henceforth a generic constant $C>0$ is independent of $k \in \mathbb{N}$. Then we have to prove that $\left\{y_{k}\right\}_{k=1}^{\infty}$ contains a subsequence which is convergent in $L^{2}\left(0, T ; W_{2}^{1}(\Omega)\right)$. Application of Lemma 2.4 to $L_{0} y_{k}=g_{k}$ yields

$$
\left\|y_{k}\right\|_{L^{2}\left(0, T ; W_{2}^{1}(\Omega)\right)} \leq C, \quad k \in \mathbb{N} .
$$


Therefore the sequence $\left\{y_{k}\right\}_{k=1}^{\infty}$ contains a subsequence which converges weakly in $L^{2}\left(0, T ; W_{2}^{1}(\Omega)\right)$ to some function $y$. Without loss of generality, we can assume that $y \equiv 0$.

Moreover we have

$$
\left\|\Delta y_{k}\right\|_{L^{2}\left(0, T ; W_{2}^{-1}(\Omega)\right)} \leq C, \quad k \in \mathbb{N} .
$$

First $\left\|g_{k}\right\|_{L^{2}(Q)} \leq C$ and $\left\|y_{k}(0, \cdot)\right\|_{W_{2}^{1}(\Omega)} \leq C$ for $k \in \mathbb{N}$. Therefore

(3.36) $\left\{g_{k}\right\}_{k=1}^{\infty}$ contains a subsequence which is weakly convergent to 0 in $L^{2}(Q)$ and $\left\{y_{k}(0, \cdot)\right\}_{k=1}^{\infty}$ contains a subsequence which is strongly convergent to 0 in $L^{2}(\Omega)$.

Let $p_{k}$ be a solution to the problem

$$
L_{0}^{*} p_{k}=-\Delta y_{k} \quad \text { in } Q,\left.\quad p_{k}\right|_{\Sigma}=0, \quad p_{k}(T, \cdot)=0 .
$$

By Lemma 2.4, the sequence $\left\{p_{k}\right\}_{k=1}^{\infty}$ is uniformly bounded in $L^{2}\left(0, T ; W_{2}^{1}(\Omega)\right) \cap$ $C\left([0, T] ; L^{2}(\Omega)\right)$.

Similarly to (2.26) and (2.27), we can prove

$$
\left\|\sum_{i=1}^{n} \frac{\partial}{\partial x_{i}}\left(b_{i} p_{k}\right)\right\|_{L^{2}\left(0, T ; W_{2}^{-1}(\Omega)\right)} \leq C \sum_{i=1}^{n}\left\|b_{i} p_{k}\right\|_{L^{2}(Q)} \leq C\left\|p_{k}\right\|_{L^{2}\left(0, T ; W_{2}^{1}(\Omega)\right)}
$$

and

$$
\left\|c p_{k}\right\|_{L^{2}\left(0, T ; W_{2}^{-1}(\Omega)\right)} \leq C\left\|p_{k}\right\|_{L^{2}\left(0, T ; W_{2}^{1}(\Omega)\right)}
$$

for all $k \in \mathbb{N}$. Therefore we see

$$
\begin{gathered}
\left\|-\sum_{i, j=1}^{n} \frac{\partial}{\partial x_{i}}\left(a_{i j} \frac{\partial p_{k}}{\partial x_{j}}\right)-\sum_{i=1}^{n} \frac{\partial}{\partial x_{i}}\left(b_{i} p_{k}\right)+c p_{k}\right\|_{L^{2}\left(0, T ; W_{2}^{-1}(\Omega)\right)} \\
\leq C\left\|p_{k}\right\|_{L^{2}\left(0, T ; W_{2}^{1}(\Omega)\right)}
\end{gathered}
$$

which implies that $\left\{\frac{\partial p_{k}}{\partial t}\right\}_{k=1}^{\infty}$ is uniformly bounded in $L^{2}\left(0, T ; W_{2}^{-1}(\Omega)\right)$ by means of

$$
\left\|L_{0}^{*} p_{k}\right\|_{L^{2}\left(0, T ; W_{2}^{-1}(\Omega)\right)} \leq C, \quad k \in \mathbb{N} .
$$

Thus, by a theorem on compactness, we can extract a subsequence $\left\{p_{k^{\prime}}\right\}_{k^{\prime}=1}^{\infty}$ such that

$$
p_{k^{\prime}} \longrightarrow p_{0} \text { in } L^{2}(Q) \text {. }
$$


Furthermore, by the uniform boundedness of $\left\|p_{k}\right\|_{C\left([0, T] ; L^{2}(\Omega)\right)}, k \in \mathbb{N}$, we see that

$$
\left\{p_{k}(0, \cdot)\right\}_{k=1}^{\infty} \text { contains a weakly convergent subsequence in } L^{2}(\Omega) .
$$

Multiplying (3.37) by $y_{k}$, taking scalar products in $L^{2}(Q)$ and integrating by parts, we obtain

$$
\int_{Q}\left|\nabla y_{k}\right|^{2} d x d t=\int_{Q} g_{k} p_{k} d x d t+\int_{\Omega} y_{k}(0, \cdot) p_{k}(0, \cdot) d x .
$$

Applying (3.36), (3.38) and (3.39) at the right side of (3.40), we complete the proof of the lemma.

Proof of Theorem 3.1. By (3.7), in terms of the mean value theorem, we can choose continuous functions $f_{0}, f_{1}, \ldots, f_{n}$ such that

$$
f\left(t, x, \zeta^{\prime}, \zeta_{0}\right)=\sum_{i=0}^{n} f_{i}\left(t, x, \zeta^{\prime}, \zeta_{0}\right) \zeta_{i} \quad(t, x) \in Q .
$$

Moreover, by (3.8), we have

$$
\left|f_{i}\left(t, x, \zeta^{\prime}, \zeta_{0}\right)\right| \leq K, \quad \forall(t, x, \zeta) \in Q \times \mathbb{R}^{n+1}, 0 \leq i \leq n .
$$

For the linear parabolic operator

$$
R(y) z=L_{0} z+\sum_{i=1}^{n} f_{i}(t, x, \nabla y, y) \frac{\partial z}{\partial x_{i}}+f_{0}(t, x, \nabla y, y) z
$$

we define the parameter $\gamma(y)$ by

$$
\begin{aligned}
\gamma(y)= & \sum_{i, j=1}^{n}\left\|a_{i j}\right\|_{W_{\infty}^{1}(Q)}+\sum_{i=1}^{n}\left(\left\|b_{i}\right\|_{L^{\infty}\left(0, T ; L^{r}(\Omega)\right)}+\left\|f_{i}(\cdot, \cdot, \nabla y, y)\right\|_{L^{\infty}\left(0, T ; L^{r}(\Omega)\right)}\right) \\
& +\|c\|_{L^{\infty}\left(0, T ; W_{r_{1}}^{-\mu}(\Omega)\right)}+\left\|f_{0}(\cdot, \cdot, \nabla y, y)\right\|_{L^{\infty}\left(0, T ; W_{r_{1}}^{-\mu}(\Omega)\right)}
\end{aligned}
$$

for every $y \in L^{2}\left(0, T ; W_{2}^{1}(\Omega)\right)$. Then by (3.8), (3.41) and (3.42), we obtain

$$
\gamma(y) \leq C,
$$

where $C>0$ is a constant independent of $y$.

Let us consider the problem of exact controllability of parabolic equations

$$
R(y) z=u+g \quad \text { in } Q, \quad u \in \mathcal{U}(\omega),
$$




$$
\left.z\right|_{\Sigma}=0, \quad z(0, x)=v_{0}(x), \quad z(T, x)=0 .
$$

By (3.43) and Lemma 3.1, we can choose $\widehat{\lambda}>0$ such that for $\lambda \geq \hat{\lambda}$, there exists $s_{0}(\lambda)$ that if $\lambda \geq \hat{\lambda}$ and $s \geq s_{0}(\lambda)$, then the problem of exact controllability (3.44) has solutions in the space $\left(Y(Q) \cap Z_{s}^{\lambda}(Q)\right) \times\left(\mathcal{U}(\omega) \cap X_{s}^{\lambda}(Q)\right)$ for all initial data $\left(v_{0}, g\right) \in \stackrel{\circ}{W_{2}^{1}}(\Omega) \times X_{s}^{\lambda}(Q)$. Moreover these solutions satisfy (3.18) where $C(\lambda, s, \gamma)>0$ is independent of $y \in L^{2}\left(0, T ; W_{2}^{1}(\Omega)\right)$.

Let us introduce mappings $\Psi: y \rightarrow \widehat{z}$ and $\Psi_{1}: y \rightarrow(\widehat{z}, \widehat{u})$ as follows: For $y \in L^{2}\left(0, T ; W_{2}^{1}(\Omega)\right)$, a pair $(\widehat{z}, \widehat{u})$ is the solution to the extremal problem:

$$
\begin{aligned}
\mathcal{J}(z, u)= & \int_{Q} e^{2 s \eta(t, x)}\left(|\nabla z|^{2}+z^{2}\right) d x d t+\int_{Q}(T-t) e^{2 s \eta(t, x)} u^{2} d x d t \rightarrow \inf \\
& R(y) z=g+u \quad \text { in } Q, \quad u \in \mathcal{U}(\omega) \\
& \left.z\right|_{\Sigma}=0, \quad z(0, x)=v_{0}(x), \quad z(T, x)=0
\end{aligned}
$$

By Lemma 3.1, for all $y \in L^{2}\left(0, T ; W_{2}^{1}(\Omega)\right)$, there exists a unique solution $(\widehat{z}, \widehat{u}) \in\left(Y(Q) \cap Z_{s}^{\lambda}(Q)\right) \times\left(\mathcal{U}(\omega) \cap X_{s}^{\lambda}(Q)\right)$ to the problem (3.45)-(3.46). Consequently the mappings $\Psi$ and $\Psi_{1}$ are well defined on the whole space $L^{2}\left(0, T ; W_{2}^{1}(\Omega)\right)$.

Let us prove that $\Psi: L^{2}\left(0, T ; W_{2}^{1}(\Omega)\right) \longrightarrow Y(Q) \cap Z_{s}^{\lambda}(Q)$ is a continuous mapping. Assume the contrary. Then there exist functions $y_{k} \in$ $L^{2}\left(0, T ; W_{2}^{1}(\Omega)\right)$ and a sequence $\left\{\left(y_{k}, \widehat{z}_{k}, \widehat{u}_{k}\right)\right\}_{k=1}^{\infty}$ satisfying $(3.47)-(3.49)$ :

$y_{k} \rightarrow y \quad$ in $L^{2}\left(0, T ; W_{2}^{1}(\Omega)\right), \quad \Psi\left(y_{k}\right)=\widehat{z_{k}} \rightarrow z \quad$ weakly $\quad$ in $Y(Q) \cap Z_{s}^{\lambda}(Q)$,

$$
\begin{gathered}
\widehat{u}_{k} \rightarrow u \quad \text { weakly } \quad \text { in } \mathcal{U}(\omega) \cap X_{s}^{\lambda}(Q) . \\
\Psi(y)=(\widehat{z}, \widehat{u}) \neq(z, u), \quad \widehat{z} \in Z_{s}^{\lambda}(Q) . \\
\text { the triple }\left(y_{k}, \widehat{z}_{k}, \widehat{u}_{k}\right) \text { satisfies }(3.46) \text { and } \\
\mathcal{J}(\widehat{z}, \widehat{u})<\mu_{0}<\mathcal{J}\left(\widehat{z}_{k}, \widehat{u}_{k}\right), \quad k \in \mathbb{N} .
\end{gathered}
$$

with some $\mu_{0}>0$.

By (3.42), (3.47) and (3.48)

$$
\begin{aligned}
& \widehat{z}\left(f_{0}\left(t, x, \nabla y_{k}, y_{k}\right)\right.\left.-f_{0}(t, x, \nabla y, y)\right)+\sum_{i=1}^{n}\left(f_{i}\left(t, x, \nabla y_{k}, y_{k}\right)\right. \\
&\left.-f_{i}(t, x, \nabla y, y)\right) \frac{\partial \widehat{z}}{\partial x_{i}} \rightarrow 0 \\
& \quad \text { in } X_{s}^{\lambda}(Q) \text { as } k \rightarrow \infty
\end{aligned}
$$


By (3.50) and Lemma 3.1, there exists a subsequence $\left\{\left(\delta_{k}, q_{k}\right)\right\}_{k=1}^{\infty} \subset(Y(Q) \cap$ $\left.Z_{s}^{\lambda}(Q)\right) \times\left(\mathcal{U}(\omega) \cap X_{s}^{\lambda}(Q)\right)$ such that

$$
\begin{aligned}
& L_{0} \delta_{k}+\sum_{i=1}^{n} f_{i}(t, x, \nabla y, y) \frac{\partial \delta_{k}}{\partial x_{i}}+f_{0}(t, x, \nabla y, y) \delta_{k} \\
& =\widehat{z}\left(f_{0}\left(t, x, \nabla y_{k}, y_{k}\right)-f_{0}(t, x, \nabla y, y)\right) \\
& \quad+\sum_{i=1}^{n}\left(f_{i}\left(t, x, \nabla y_{k}, y_{k}\right)-f_{i}(t, x, \nabla y, y)\right) \frac{\partial \widehat{z}}{\partial x_{i}}+q_{k} \quad \text { in } Q \\
& \left.\quad \delta_{k}\right|_{\Sigma}=0, \quad \delta_{k}(0, x)=\delta_{k}(T, x)=0, \quad q_{k} \in U(\omega), \\
& \left\|\delta_{k}\right\|_{Y(Q) \cap Z_{s}^{\lambda}(Q)}+\left\|q_{k}\right\|_{X_{s}^{\lambda}(Q)} \rightarrow 0 \quad \text { as } k \rightarrow \infty .
\end{aligned}
$$

We set

$$
\tilde{z}_{k}=\widehat{z}-\delta_{k}, \quad \tilde{u}_{k}=\widehat{u}-q_{k} .
$$

By (3.51) and (3.52), the following holds:

$$
\begin{gathered}
L_{0} \tilde{z}_{k}+\sum_{i=1}^{n} f_{i}\left(t, x, \nabla y_{k}, y_{k}\right) \frac{\partial \tilde{z}_{k}}{\partial x_{i}}+f_{0}\left(t, x, \nabla y_{k}, y_{k}\right) \tilde{z}_{k} \\
=g+\tilde{u}_{k} \quad \text { in } Q, \quad \tilde{u}_{k} \in \mathcal{U}(\omega), \\
\left.\tilde{z}_{k}\right|_{\Sigma}=0, \quad \tilde{z}_{k}(0, x)=v_{0}(x), \quad \tilde{z}_{k}(T, x)=0 .
\end{gathered}
$$

Moreover, by (3.53),

$$
\lim _{k \rightarrow \infty} \mathcal{J}\left(\tilde{z}_{k}, \tilde{u}_{k}\right)=\mathcal{J}(\widehat{z}, \widehat{u}) .
$$

By (3.55) and (3.56), the pair $\left(\tilde{z}_{k}, \tilde{u}_{k}\right)$ is an admissible element of the extremal problem (3.45)-(3.46). Therefore by the definition of the mapping $\Psi_{1}$, we obtain

$$
\mathcal{J}\left(\widehat{z}_{k}, \widehat{u}_{k}\right) \leq \mathcal{J}\left(\tilde{z}_{k}, \tilde{u}_{k}\right), \quad k \in \mathbb{N} .
$$

Now (3.57) and (3.58) contradict (3.49). Thus the continuity of $\Psi$ is proved.

Denote by $B_{r}$ the ball in $L^{2}\left(0, T ; W_{2}^{1}(\Omega)\right)$ with the radius $r$ and the centre at zero. By (3.18) and (3.43), if $s>0$ is sufficiently large, then, for all sufficiently large $r$, we obtain

$$
\Psi\left(B_{r}\right) \subset B_{r}
$$

Moreover, if $\mathfrak{S}$ is a bounded set in $L^{2}\left(0, T ; W_{2}^{1}(\Omega)\right)$, then by $(3.18)$ the set $\Psi \mathfrak{S}$ is bounded in $Y(Q)$. Since, by Lemma 3.3 , the imbedding $Y(Q) \subset$ 
$L^{2}\left(0, T ; W_{2}^{1}(\Omega)\right)$ is compact, the mapping $\Psi$ from $L^{2}\left(0, T ; W_{2}^{1}(\Omega)\right)$ to itself is compact.

Applying the Schauder fixed point theorem, we find that there exists a fixed point $y$ of the mapping $\Psi$ :

$$
\Psi(y)=y .
$$

Obviously a pair $\Psi_{1}(y)=(y, u)$ is a solution to (3.1)-(3.2) with $v(T, x)=0$, $x \in \Omega$.

Finally we state the global exact zero controllability by boundary control.

Theorem 3.2. Let $v_{0} \in W_{2}^{1}(\Omega), v_{1} \equiv 0$, and let conditions (2.3)-(2.4), (3.7) and (3.8) be fulfilled. Then there exists $\widehat{\lambda}>0$ such that for $\lambda \geq \hat{\lambda}$, there exists a constant $s_{0}(\lambda)$ such that if $g \in X_{s}^{\lambda}(Q)$ with $\lambda \geq \hat{\lambda}$ and $s \geq s_{0}(\lambda)$, then there exists a solution pair $(y, u) \in Y(Q) \times L^{2}\left(0, T ; H^{\frac{1}{2}}(\partial \Omega)\right)$ of the problem (3.5)-(3.6).

The proof of Theorem 3.2 is done by applying the argument in the proof of Theorem 3.3 from [25] on the basis of Theorem 3.1. We omit the details.

\section{Appendix I}

Proof of Lemma 2.2. The proof for $n=1,2$ is similar to the case of $n \geq 3$, and we give the proof only for the case of $n \geq 3$. Henceforth we set $\Delta_{h} u=u(x+h)-u(x), x \in \mathbb{R}^{n}$. Then we have

$$
\|u\|_{W_{p}^{\mu}\left(\mathbb{R}^{n}\right)}=\left\{\|u\|_{L^{p}\left(\mathbb{R}^{n}\right)}^{p}+\int_{\mathbb{R}^{n}} \frac{1}{|h|^{n+\mu p}}\left\|\Delta_{h} u\right\|_{L^{p}\left(\mathbb{R}^{n}\right)}^{p} d h\right\}^{\frac{1}{p}},
$$

where $0<\mu<1 / 2,1<p<\infty$. By the smoothness of $\partial \Omega$, for the proof, instead of a function $v$, we can consider its extension in $\mathbb{R}^{n}$ such that

$\|v\|_{W_{2}^{l}\left(\mathbb{R}^{n}\right)} \leq C\|v\|_{W_{2}^{l}(\Omega)}, \quad \forall l \in\left[0, \frac{1}{2}\right] ; \quad\|v\|_{W_{2}^{\ell}\left(\mathbb{R}^{n}\right)} \leq C\|v\|_{W_{2}^{\ell}(\Omega)}, \quad \forall \ell \in[0,1]$.

By $B_{p, q}^{\mu}\left(\mathbb{R}^{n}\right)$, we denote the Besov space (e.g., Triebel [54]). Henceforth, taking these extensions of functions under consideration, we identify $\|\cdot\|_{B_{p, q}^{\mu}\left(\mathbb{R}^{n}\right)}$ with $\|\cdot\|_{B_{p, q}^{\mu}(\Omega)}$ (e.g., Theorem 4.2.2 in [54]). Since $v \in \stackrel{\circ}{W_{2}^{1}}(\Omega)$ and $z \in W_{2}^{\frac{1}{2}-\delta}(\Omega)$, we take the 0-extensions, so that we regard $v \in W_{2}^{1}\left(\mathbb{R}^{n}\right)$ and $z \in W_{2}^{\frac{1}{2}-\delta}\left(\mathbb{R}^{n}\right)$. By the definition, we have

$$
\|z v\|_{W_{r_{1}^{\prime}}^{\mu}(\Omega)}^{2} \leq 2\|z v\|_{L^{r_{1}^{\prime}(\Omega)}}^{2}+2\left(\int_{\mathbb{R}^{n}} \frac{\left\|\Delta_{h}(z v)\right\|_{L_{1}^{r_{1}^{\prime}\left(\mathbb{R}^{n}\right)}}^{r^{\prime}}}{|h|^{n+\mu r_{1}^{\prime}}} d h\right)^{\frac{2}{r_{1}^{\prime}}}
$$




$$
\leq 2\|z v\|_{L^{r_{1}^{\prime}(\Omega)}}^{2}+C\left(\int_{\mathbb{R}^{n}} \frac{\left\|v \Delta_{h} z\right\|_{L_{1}^{r_{1}^{\prime}}}^{r_{\left.\mathbb{R}^{n}\right)}^{\prime}}+\left\|z \Delta_{h} v\right\|_{L^{r_{1}^{\prime}}}^{r_{\left.\mathbb{R}^{n}\right)}^{\prime}}}{|h|^{n+\mu r_{1}^{\prime}}} d h\right)^{\frac{2}{r_{1}^{\prime}}} .
$$

We set

$$
\kappa=\frac{2 n r_{1}^{\prime}}{2 n-n r_{1}^{\prime}+2 r_{1}^{\prime}}, \quad \kappa_{1}=\frac{2 n r_{1}^{\prime}}{2 n-n r_{1}^{\prime}+(1-2 \delta) r_{1}^{\prime}} .
$$

We fix $\delta \in(0, \mu)$. Here and henceforth, $C>0$ denotes a generic constant which is independent of $z$ and $v$. Obviously $2 n /(n-1+2 \delta) r^{\prime}>1$. Using Theorem 2.5.1 in [54] and the Hölder inequality, we obtain

$$
\begin{aligned}
\|z v\|_{W_{r_{1}^{\prime}}^{\mu}(\Omega)}^{2} \leq & C\|z\|_{L^{\kappa}(\Omega)}^{2}\|v\|_{L^{\frac{2 n}{n-2}}(\Omega)}^{2} \\
& +C\left(\int_{\mathbb{R}^{n}} \frac{\left\|\Delta_{h} z\right\|_{L^{\kappa}(\Omega)}^{r^{\prime}}\|v\|_{L^{\frac{2 n}{n-2}}(\Omega)}^{r_{1}^{\prime}}}{|h|^{n+\mu r_{1}^{\prime}}} d h\right)^{\frac{2}{r_{1}^{\prime}}} \\
& +C\left(\int_{\mathbb{R}^{n}} \frac{\left\|\Delta_{h} v\right\|_{L^{\kappa}(\Omega)}^{r_{1}^{\prime}}\|z\|_{L^{\frac{2 n}{n-1+2 \delta}}(\Omega)}^{r_{1}^{\prime}}}{|h|^{n+\mu r_{1}^{\prime}}}\right)^{\frac{2}{r_{1}^{\prime}}} \\
\leq & \left.C\left(\|z\|_{W_{2}^{\frac{1}{2}-\delta}(\Omega)}^{2}\|v\|_{W_{2}^{1}(\Omega)}^{2}\right)+\|z\|_{B_{\kappa, r_{1}^{\prime}}^{\mu}(\Omega)}^{2}\|v\|_{W_{2}^{1}(\Omega)}^{2}+\|z\|_{W_{2}^{\frac{1}{2}-\delta}(\Omega)}^{2}\|v\|_{B_{\kappa_{1}, r_{1}^{\prime}}^{\mu}(\Omega)}^{2}\right) .
\end{aligned}
$$

Here we have also used

$$
W_{2}^{1}(\Omega) \subset L^{\frac{2 n}{n-2}}(\Omega), \quad W_{2}^{\frac{1}{2}-\delta}(\Omega) \subset L^{\frac{2 n}{n-1+2 \delta}}(\Omega)
$$

and

$$
W_{2}^{\frac{1}{2}-\delta}(\Omega) \subset L^{\kappa}(\Omega)
$$

which are true by the Sobolev imbedding (e.g., [1]) and $0<\delta<\mu$,

$$
r_{1}^{\prime}<\frac{2 n}{2 n-3+2 \mu}
$$

Henceforth, by (1), we can set

$$
\frac{n}{r_{1}^{\prime}}=n-\frac{3}{2}+\mu+\varepsilon
$$

with some $\varepsilon>0$. On the other hand, we can prove

$$
W_{2}^{\frac{1}{2}-\delta}(\Omega) \subset B_{\kappa, r_{1}^{\prime}}^{\mu}(\Omega)
$$


and

$$
W_{2}^{1}(\Omega) \subset B_{\kappa_{1}, r_{1}^{\prime}}^{\mu}(\Omega)
$$

Proof of (3). We can take sufficiently small $\delta>0$. By the imbedding of Besov spaces (e.g., Triebel [54, Theorem 2.3.2 (c)]), noting $r_{1}>1$, we have

$$
W_{2}^{\frac{1}{2}-\delta}\left(\mathbb{R}^{n}\right) \subset B_{2,2}^{\frac{1}{2}-\delta}\left(\mathbb{R}^{n}\right) \subset B_{2,1}^{\frac{1}{2}-2 \delta}\left(\mathbb{R}^{n}\right) \subset B_{2, r_{1}^{\prime}}^{\frac{1}{2}-2 \delta}\left(\mathbb{R}^{n}\right) .
$$

By (2), we can easily verify that $\frac{1}{2}-2 \delta-\frac{n}{2} \geq \mu-\frac{n}{\kappa}$ and $\mu<\frac{1}{2}-2 \delta$ if we take sufficiently small $\delta>0$ such that $0<\delta<\min \left\{\frac{\varepsilon}{2}, \frac{1}{2}\left(\frac{1}{2}-\mu\right)\right\}$. Therefore, by the imbedding of Besov spaces (e.g., Triebel [54, Theorem 4.6.2]), we can see

$$
B_{2, r_{1}^{\prime}}^{\frac{1}{2}-2 \delta}(\Omega) \subset B_{\kappa, r_{1}^{\prime}}^{\mu}(\Omega)
$$

Thus (5) implies (3).

Proof of (4). Similarly we can see

$$
W_{2}^{1}\left(\mathbb{R}^{n}\right) \subset B_{2,2}^{1}\left(\mathbb{R}^{n}\right) \subset B_{2,1}^{1-\delta_{1}}\left(\mathbb{R}^{n}\right) \subset B_{2, r_{1}^{\prime}}^{1-\delta_{1}}\left(\mathbb{R}^{n}\right)
$$

for any small $\delta_{1}>0$ (e.g., [54, Theorem 2.3.2 (c)]). By (2), we can see $1-\delta_{1}-$ $\frac{n}{2} \geq \mu-\frac{n}{\kappa_{1}}$ and $\mu<1-\delta_{1}$ if $0<\delta<\varepsilon$ and $0<\delta_{1}<\min \{\varepsilon-\delta, 1-\mu\}$. Then we obtain

$$
B_{2, r_{1}^{\prime}}^{1-\delta_{1}}(\Omega) \subset B_{\kappa_{1}, r_{1}^{\prime}}^{\mu}(\Omega)
$$

with which we combine (6) to obtain (4).

In view of (3) and (4), we have

$$
\|z\|_{B_{\kappa, r_{1}^{\prime}}^{\mu}(\Omega)} \leq C\|z\|_{W_{2}^{\frac{1}{2}-\delta}(\Omega)}
$$

and

$$
\|v\|_{B_{\kappa_{1}, r_{1}^{\prime}}^{\mu}(\Omega)} \leq C\|v\|_{W_{2}^{1}(\Omega)}
$$

where $C=C\left(\Omega, \mu, \kappa, \kappa_{1}, r_{1}^{\prime}, \delta\right)>0$ is independent of $z$. Thus the proof of Lemma 2.2 is complete.

Remark. In terms of the Triebel-Lizorkin space, we can give the following concise proof: First we extend the functions $z, v$ by zero on $\mathbb{R}^{n}$. We introduce the Triebel-Lizorkin space $F_{p, q}^{s}$ (e.g., p. 8 in Runst and Sickel [47]). Then we note that $W_{p}^{s}\left(\mathbb{R}^{n}\right)=F_{p, p}^{s}$. Now we show that the statement of this lemma follows from the general embedding theorem proved in [47, p. 189]. Let 
us check the conditions of that theorem in the case of $n \geq 3$. For the case of $n=1,2$, the proof is similar and omitted. We set $s=\mu, p=q=r_{1}^{\prime}$, $p_{1}=p_{2}=q_{1}=q_{2}=2$. Let $s_{1}=\frac{1}{2}-\delta$ and $s_{2}=1$. There we choose the parameter $\delta \in\left(0, \frac{1}{2}\right)$ such that $s<s_{1}<s_{2}$. Obviously $\frac{1}{p}=1-\frac{1}{r_{1}} \leq \sum_{j=1}^{2} \frac{1}{p_{j}}=1$ and $n>\sum_{j=1}^{2}\left(\frac{n}{p_{j}}-s_{j}\right)=n-\frac{3}{2}+\delta$. Finally, by the condition on $r_{1}$, there exists $\varepsilon>0$ such that $\frac{n}{p}-s=n-\frac{n}{r_{1}}-\mu=n-\frac{3}{2}+\varepsilon>\sum_{j=1}^{2}\left(\frac{n}{p_{j}}-s_{j}\right)=n-\frac{3}{2}+\delta$ provided that $\delta<\varepsilon$. Hence we have $F_{p_{1}, q_{1}}^{s_{1}} \cdot F_{p_{2}, q_{2}}^{s_{2}} \subset F_{p, q}^{s}$, that is, $F_{2,2}^{\frac{1}{2}-\delta} \cdot F_{2,2}^{1} \subset F_{r_{1}^{\prime}, r_{1}^{\prime}}^{\mu}$. The proof of this lemma is complete.

\section{Appendix II}

Proof of Lemma 2.3 in the case of $d=0$. The proof is similar to the proof in [4], [25] where $a_{i j} \in C^{1,2}(\bar{\Omega}), 1 \leq i, j \leq n$. Let us consider the operator

$$
\widehat{L} y=\frac{\partial y}{\partial t}-\sum_{i, j=1}^{n} a_{i j}(t, x) \frac{\partial^{2} y}{\partial x_{i} \partial x_{j}} .
$$

We set

$$
\widehat{c}(t, x)=c(t, x)+\sum_{i=1}^{n} \frac{\partial b_{i}}{\partial x_{i}}(t, x)
$$

and

(2) $\tilde{g}(t, x)=\left(g(t, x)-\sum_{i=1}^{n} b_{i}(t, x) \frac{\partial y}{\partial x_{i}}-\widehat{c}(t, x) y+\sum_{i, j=1}^{n} \frac{\partial a_{i j}(t, x)}{\partial x_{i}} \frac{\partial y}{\partial x_{j}}\right)$.

We denote $w(t, x)=e^{s \alpha} y(t, x)$. By (2.7), we have

$$
w(T, \cdot)=w(0, \cdot)=0 \quad \text { in } \quad \Omega .
$$

We define an operator $P$ by

$$
P w=e^{s \alpha} \widehat{L}\left(e^{-s \alpha} w\right)
$$

It follows from (2.1) and (1), (2) that

$$
P w=e^{s \alpha} \widetilde{g} \quad \text { in } \quad Q .
$$

We notice that the operator $P$ can be written explicitly as follows

$$
P w=\frac{\partial w}{\partial t}-\sum_{i, j=1}^{n} a_{i j} \frac{\partial^{2} w}{\partial x_{i} \partial x_{j}}+2 s \lambda \varphi \sum_{i, j=1}^{n} a_{i j} \psi_{x_{i}} \frac{\partial w}{\partial x_{j}}+s \lambda^{2} \varphi a(t, x, \nabla \psi, \nabla \psi) w
$$




$$
-s^{2} \lambda^{2} \varphi^{2} a(t, x, \nabla \psi, \nabla \psi) w+s \lambda \varphi w \sum_{i, j=1}^{n} a_{i j} \psi_{x_{i} x_{j}}-s \frac{\partial \alpha}{\partial t} w
$$

Here and henceforth, we set $\psi_{x_{i}}=\frac{\partial \psi}{\partial x_{i}}, \psi_{x_{i} x_{j}}=\frac{\partial^{2} \psi}{\partial x_{i} \partial x_{j}}, 1 \leq i, j \leq n$. We recall that the quadratic form $a(t, x, \xi, \eta)$ was defined in (2.4). We further introduce the operators $L_{1}, L_{2}$ as follows:

$$
\begin{aligned}
L_{1} w & =-\sum_{i, j=1}^{n} a_{i j} \frac{\partial^{2} w}{\partial x_{i} \partial x_{j}}-\lambda^{2} s^{2} \varphi^{2} a(t, x, \nabla \psi, \nabla \psi) w-s \frac{\partial \alpha}{\partial t} w \\
L_{2} w & =\frac{\partial w}{\partial t}+2 s \lambda \varphi \sum_{i, j=1}^{n} a_{i j} \psi_{x_{i}} \frac{\partial w}{\partial x_{j}}+2 s \lambda^{2} \varphi a(t, x, \nabla \psi, \nabla \psi) w .
\end{aligned}
$$

It follows from (2), (6), (7) and (8) that

$$
L_{1} w+L_{2} w=f_{s} \quad \text { in } \quad Q
$$

where

$$
f_{s}(t, x)=\widetilde{g} e^{s \alpha}-s \lambda \varphi w \sum_{i, j=1}^{n} a_{i j} \psi_{x_{i} x_{j}}+s \lambda^{2} \varphi a(t, x, \nabla \psi, \nabla \psi) w .
$$

Taking $L_{2}$-norms of the both sides of (9), we obtain

$$
\left\|f_{s}\right\|_{L^{2}(Q)}^{2}=\left\|L_{1} w\right\|_{L^{2}(Q)}^{2}+\left\|L_{2} w\right\|_{L^{2}(Q)}^{2}+2\left(L_{1} w, L_{2} w\right)_{L^{2}(Q)} .
$$

By (7) and (8), we have the following equality:

$$
\begin{aligned}
\left(L_{1} w, L_{2} w\right)_{L^{2}(Q)} & \\
= & \left(-\sum_{i, j=1}^{n} a_{i j} \frac{\partial^{2} w}{\partial x_{i} \partial x_{j}}-\lambda^{2} s^{2} \varphi^{2} a(t, x, \nabla \psi, \nabla \psi) w\right. \\
& \left.-s \frac{\partial \alpha}{\partial t} w, \frac{\partial w}{\partial t}+2 s \lambda^{2} \varphi a(t, x, \nabla \psi, \nabla \psi) w\right)_{L^{2}(Q)} \\
& -\int_{Q}\left(2 \lambda^{3} s^{3} \varphi^{3} a(t, x, \nabla \psi, \nabla \psi) w+2 s^{2} \lambda \varphi \frac{\partial \alpha}{\partial t} w\right) a(t, x, \nabla \psi, \nabla w) d x d t \\
& -\int_{Q}\left(\sum_{i, j=1}^{n} a_{i j} \frac{\partial^{2} w}{\partial x_{i} \partial x_{j}}\right) 2 s \lambda \varphi a(t, x, \nabla \psi, \nabla w) d x d t .
\end{aligned}
$$


Integrating by parts in the first term of the right-hand side of (11), we obtain

$$
\begin{aligned}
A_{0}= & \left(-\sum_{i, j=1}^{n} a_{i j} \frac{\partial^{2} w}{\partial x_{i} \partial x_{j}}-\lambda^{2} s^{2} \varphi^{2} a(t, x, \nabla \psi, \nabla \psi) w\right. \\
& \left.\quad-s w \frac{\partial \alpha}{\partial t}, \frac{\partial w}{\partial t}+2 s \lambda^{2} \varphi a(t, x, \nabla \psi, \nabla \psi) w\right)_{L^{2}(Q)} \\
= & \int_{Q}\left(\frac{\partial w}{\partial t} \sum_{i, j=1}^{n} \frac{\partial a_{i j}}{\partial x_{j}} \frac{\partial w}{\partial x_{i}}+\sum_{i, j=1}^{n} a_{i j} \frac{\partial w}{\partial x_{i}} \frac{\partial w_{t}}{\partial x_{j}}\right. \\
& -\frac{\lambda^{2} s^{2} \varphi^{2}}{2} a(t, x, \nabla \psi, \nabla \psi) \frac{\partial w^{2}}{\partial t}-\frac{s}{2} \frac{\partial \alpha}{\partial t} \frac{\partial w^{2}}{\partial t}-2 s^{3} \varphi^{3} \lambda^{4} a(t, x, \nabla \psi, \nabla \psi)^{2} w^{2} \\
& -2 s^{2} \lambda^{2} \frac{\partial \alpha}{\partial t} \varphi a(t, x, \nabla \psi, \nabla \psi) w^{2}+2 \lambda^{2} s \varphi a(t, x, \nabla \psi, \nabla \psi) w \sum_{i, j=1}^{n} \frac{\partial a_{i j}}{\partial x_{j}} \frac{\partial w}{\partial x_{i}} \\
& +2 s \lambda^{2} \varphi a(t, x, \nabla \psi, \nabla \psi) a(t, x, \nabla w, \nabla w) \\
& \left.+2 s \lambda^{2} w \sum_{i, j=1}^{n} a_{i j} \frac{\partial w}{\partial x_{j}} \frac{\partial}{\partial x_{i}}(\varphi a(t, x \nabla \psi, \nabla \psi))\right) d x d t .
\end{aligned}
$$

Integrating by parts in the second term of the right-hand side of (11), we have

$$
\begin{aligned}
&-\int_{Q}\left(2 \lambda^{3} s^{3} w \varphi^{3} a(t, x, \nabla \psi, \nabla \psi) a(t, x, \nabla \psi, \nabla w)\right. \\
&+\left.2 s^{2} \lambda \frac{\partial \alpha}{\partial t} w \varphi a(t, x, \nabla \psi, \nabla w)\right) d x d t \\
&=-\int_{Q}\left(\lambda^{3} s^{3} \varphi^{3} a(t, x, \nabla \psi, \nabla \psi) a\left(t, x, \nabla \psi, \nabla w^{2}\right)\right. \\
&\left.+s^{2} \frac{\partial \alpha}{\partial t} \varphi \lambda a\left(t, x, \nabla \psi, \nabla w^{2}\right)\right) d x d t \\
&= \int_{Q}\left(3 \lambda^{4} s^{3} \varphi^{3} a(t, x, \nabla \psi, \nabla \psi)^{2} w^{2}\right. \\
&+w^{2} \varphi^{3} \lambda^{3} s^{3} \sum_{i, j=1}^{n} \frac{\partial}{\partial x_{i}}\left(a_{i j} \psi x_{j} a(t, x, \nabla \psi, \nabla \psi)\right) \\
&\left.+\sum_{i, j=1}^{n} \frac{\partial}{\partial x_{j}}\left(\frac{s^{2} \lambda^{2} \varphi}{2} a_{i j} \frac{\partial \psi}{\partial x_{i}} \frac{\partial \alpha}{\partial t}\right) w^{2}\right\} d x d t .
\end{aligned}
$$


Finally, integrating by parts the third term of the right-hand side of (11) and taking into account (2.5), we have

$$
\begin{aligned}
& A_{1}=\int_{Q}-\left(\sum_{i, j=1}^{n} a_{i j} \frac{\partial^{2} w}{\partial x_{i} \partial x_{j}}\right)\left(2 s \lambda \varphi \sum_{k, \ell=1}^{n} a_{k \ell} \psi_{x_{k}} \frac{\partial w}{\partial x_{\ell}}\right) d x d t \\
& =\int_{Q}\left(\sum_{i, j=1}^{n} \frac{\partial a_{i j}}{\partial x_{j}} \frac{\partial w}{\partial x_{i}} 2 s \lambda \varphi \sum_{k, \ell=1}^{n} a_{k \ell} \psi_{x_{k}} \frac{\partial w}{\partial x_{\ell}}+2 s \lambda^{2} \varphi a(t, x, \nabla \psi, \nabla w)^{2}\right. \\
& +2 s \lambda \varphi \sum_{i, j=1}^{n}\left(a_{i j} \frac{\partial w}{\partial x_{i}} \sum_{k, \ell=1}^{n} \frac{\partial}{\partial x_{j}}\left(a_{k \ell} \psi_{x_{k}}\right) \frac{\partial w}{\partial x_{\ell}}\right) \\
& \left.+2 s \lambda \varphi \sum_{i, j=1}^{n} a_{i j} \frac{\partial w}{\partial x_{i}} \sum_{k, \ell=1}^{n} a_{k \ell} \psi_{x_{k}} \frac{\partial^{2} w}{\partial x_{j} \partial x_{\ell}}\right) d x d t \\
& +\int_{\Sigma} 2 s \lambda \varphi|\nabla \psi|\left|\frac{\partial w}{\partial \nu_{A}}\right|^{2} d \Sigma \\
& =\iint_{Q} \sum_{i, j=1}^{n} \frac{\partial a_{i j}}{\partial x_{j}} \frac{\partial w}{\partial x_{i}} 2 s \lambda \varphi \sum_{k, \ell=1}^{n} a_{k \ell} \psi_{x_{k}} \frac{\partial w}{\partial x_{\ell}}+2 s \lambda^{2} \varphi a(t, x, \nabla \psi, \nabla w)^{2} \\
& +2 s \lambda \varphi \sum_{i, j=1}^{n}\left(a_{i j} \frac{\partial w}{\partial x_{i}} \sum_{k, \ell=1}^{n} \frac{\partial}{\partial x_{j}}\left(a_{k \ell} \psi_{x_{k}}\right) \frac{\partial w}{\partial x_{\ell}}\right) \\
& -s \lambda \varphi \sum_{k, \ell=1}^{n} a_{k \ell} \psi_{x_{k}} \sum_{i, j=1}^{n} \frac{\partial a_{i j}}{\partial x_{\ell}} \frac{\partial w}{\partial x_{i}} \frac{\partial w}{\partial x_{j}} \\
& \left.+s \lambda \varphi \sum_{k, \ell=1}^{n} a_{k \ell} \psi_{x_{k}} \frac{\partial}{\partial x_{\ell}} \sum_{i, j=1}^{n} a_{i j} \frac{\partial w}{\partial x_{i}} \frac{\partial w}{\partial x_{j}}\right\} d x d t \\
& +\int_{\Sigma} 2 s \lambda \varphi|\nabla \psi|\left|\frac{\partial w}{\partial \nu_{A}}\right|^{2} d \Sigma \text {. }
\end{aligned}
$$

Integrating by parts once again, we obtain

$$
\begin{aligned}
A_{1}= & \int_{Q}\left\{\sum_{i, j=1}^{n} \frac{\partial a_{i j}}{\partial x_{j}} \frac{\partial w}{\partial x_{i}} 2 s \lambda \varphi \sum_{k, \ell=1}^{n} a_{k \ell} \psi_{x_{k}} \frac{\partial w}{\partial x_{\ell}}+2 s \lambda^{2} \varphi a(t, x, \nabla \psi, \nabla w)^{2}\right. \\
& +2 s \lambda \varphi \sum_{i, j=1}^{n}\left(a_{i j} \frac{\partial w}{\partial x_{i}} \sum_{k, \ell=1}^{n} \frac{\partial}{\partial x_{j}}\left(a_{k \ell} \psi_{x_{k}}\right) \frac{\partial w}{\partial x_{\ell}}\right)
\end{aligned}
$$




$$
\begin{aligned}
& -s \lambda \varphi \sum_{k, \ell=1}^{n} a_{k \ell} \psi_{x_{k}} \sum_{i, j=1}^{n} \frac{\partial a_{i j}}{\partial x_{\ell}} \frac{\partial w}{\partial x_{i}} \frac{\partial w}{\partial x_{j}} \\
& -s \lambda^{2} \varphi a(t, x, \nabla \psi, \nabla \psi) a(t, x, \nabla w, \nabla w) \\
& \left.-a(t, x, \nabla w, \nabla w) s \lambda \varphi \sum_{k, \ell=1}^{n} \frac{\partial}{\partial x_{\ell}}\left(a_{k \ell} \psi_{x_{k}}\right)\right\} d x d t \\
& +\int_{\Sigma} s \lambda \varphi|\nabla \psi|\left|\frac{\partial w}{\partial \nu_{A}}\right|^{2} d \Sigma,
\end{aligned}
$$

where we used the fact: $\nu=-\nabla \psi /|\nabla \psi|$ which is seen from $\psi_{\mid \partial \Omega}=0$.

By virtue of (12), (13) and (15), one can rewrite (11) as follows.

$$
\begin{aligned}
& \left(L_{1} w, L_{2} w\right)_{L^{2}(Q)} \\
& =\int_{Q}\left\{\lambda^{4} s^{3} \varphi^{3} a(t, x, \nabla \psi, \nabla \psi)^{2} w^{2}\right. \\
& \quad+s \lambda^{2} \varphi a(t, x, \nabla \psi, \nabla \psi) a(t, x, \nabla w, \nabla w)+L_{2} w \sum_{i, j=1}^{n} \frac{\partial a_{i j}}{\partial x_{j}} \frac{\partial w}{\partial x_{i}} \\
& \left.\quad+2 s \lambda^{2} \varphi a(t, x, \nabla \psi, \nabla w)^{2}\right\} d x d t \\
& \quad+\int_{\Sigma} s \lambda \varphi|\nabla \psi|\left|\frac{\partial w}{\partial \nu_{A}}\right|^{2} d \Sigma+X_{1},
\end{aligned}
$$

where we put

$$
\begin{aligned}
X_{1}= & \int_{Q}\left\{2 s \lambda^{2} w \sum_{i, j=1}^{n} a_{i j} \frac{\partial w}{\partial x_{j}} \frac{\partial}{\partial x_{i}}(\varphi a(t, x, \nabla \psi, \nabla \psi))\right. \\
& +\frac{1}{2} \frac{\partial}{\partial t}\left(\lambda^{2} s^{2} \varphi^{2} a(t, x, \nabla \psi, \nabla \psi)\right) w^{2}-\frac{s w^{2}}{2} \frac{\partial^{2} \alpha}{\partial t^{2}} \\
& +2 s \lambda \varphi \sum_{i, j=1}^{n}\left(a_{i j} \frac{\partial w}{\partial x_{i}} \sum_{k, \ell=1}^{n} \frac{\partial\left(a_{k \ell} \psi_{x_{k}}\right)}{\partial x_{j}} \frac{\partial w}{\partial x_{\ell}}\right) \\
& -s \lambda \varphi \sum_{k, \ell=1}^{n} a_{k \ell} \psi_{x_{k}} \sum_{i, j=1}^{n} \frac{\partial a_{i j}}{\partial x_{\ell}} \frac{\partial w}{\partial x_{i}} \frac{\partial w}{\partial x_{j}} \\
& -a(t, x, \nabla w, \nabla w) s \lambda \varphi \sum_{k, \ell=1}^{n} \frac{\partial}{\partial x_{\ell}}\left(a_{k \ell} \psi_{x_{k}}\right)
\end{aligned}
$$




$$
\begin{aligned}
& -\frac{1}{2} \sum_{i, j=1}^{n} \frac{\partial a_{i j}}{\partial t} \frac{\partial w}{\partial x_{i}} \frac{\partial w}{\partial x_{j}}+w^{2} \varphi^{3} \lambda^{3} s^{3} \sum_{i, j=1}^{n} \frac{\partial}{\partial x_{i}}\left(a_{i j} \psi_{x_{j}} a(t, x, \nabla \psi, \nabla \psi)\right) \\
& \left.-\sum_{i, j=1}^{n} \frac{\partial}{\partial x_{j}}\left(\frac{s^{2} \varphi \lambda^{2}}{2} a_{i j} \frac{\partial \psi}{\partial x_{i}} \frac{\partial \alpha}{\partial t}\right) w^{2}\right\} d x d t .
\end{aligned}
$$

Hence we can easily prove

$$
\begin{gathered}
\left|X_{1}\right| \leq C \int_{Q}\left(\left(s^{3} \lambda^{3} \varphi^{3}+s^{2} \lambda^{4} \varphi^{3}\right) w^{2}+(s \lambda \varphi+1)|\nabla w|^{2}\right) d x d t \\
s \geq 1, \quad \lambda \geq 1 .
\end{gathered}
$$

Here and henceforth $C>0$ denotes a generic constant which is independent of $s$ and $\lambda$.

Therefore, by virtue of (10) and (16), we have

$$
\begin{aligned}
\left\|f_{s}\right\|_{L^{2}(Q)}^{2}= & \left\|L_{1} w\right\|_{L^{2}(Q)}^{2}+\left\|L_{2} w\right\|_{L^{2}(Q)}^{2}+2 \int_{Q}\left(\lambda^{4} s^{3} \varphi^{3} a(t, x, \nabla \psi, \nabla \psi)^{2} w^{2}\right. \\
& +s \lambda^{2} \varphi a(t, x, \nabla \psi, \nabla \psi) a(t, x, \nabla w, \nabla w) \\
& \left.+\left(L_{2} w\right)\left(\sum_{i, j=1}^{n} \frac{\partial a_{i j}}{\partial x_{j}} \frac{\partial w}{\partial x_{i}}\right)+2 s \lambda^{2} \varphi a(t, x, \nabla \psi, \nabla w)^{2}\right) d x d t \\
& +\int_{\Sigma} s \lambda|\nabla \psi|^{2} \varphi\left|\frac{\partial w}{\partial \nu_{A}}\right|^{2} d \Sigma+X_{1}
\end{aligned}
$$

Applying the Cauchy-Bunyakovskii inequality in (18), we obtain

$$
\begin{aligned}
\left\|L_{1} w\right\|_{L^{2}(Q)}^{2}+ & \frac{1}{2}\left\|L_{2} w\right\|_{L^{2}(Q)}^{2} \\
+ & 2 \int_{Q}\left(\lambda^{4} s^{3} \varphi^{3} a(t, x, \nabla \psi, \nabla \psi)^{2} w^{2}\right. \\
& +s \lambda^{2} \varphi a(t, x, \nabla \psi, \nabla \psi) a(t, x, \nabla w, \nabla w) \\
& \left.-4\left(\sum_{i, j=1}^{n} \frac{\partial a_{i j}}{\partial x_{j}} \frac{\partial w}{\partial x_{i}}\right)^{2}\right) d x d t+X_{1} \leq\left\|f_{s}\right\|_{L^{2}(Q)}^{2} .
\end{aligned}
$$

We recall that by Lemma 2.1

$$
|\nabla \psi(x)|>\kappa>0, \quad \forall x \in \overline{\Omega \backslash \omega_{0}} .
$$


Hence, taking a parameter $\lambda>\widehat{\lambda}>0$ sufficiently large in (19), by virtue of (17), we obtain: There exists $s_{0}(\lambda)>0$ such that

$$
\begin{gathered}
\left\|L_{1} w\right\|_{L^{2}(Q)}^{2}+\frac{1}{2}\left\|L_{2} w\right\|_{L^{2}(Q)}^{2}+\int_{Q}\left(\lambda^{4} s^{3} \varphi^{3} w^{2}+s \lambda^{2} \varphi|\nabla w|^{2}\right) d x d t \\
\leq C\left(\int_{Q_{\omega}}\left(\lambda^{4} s^{3} \varphi^{3} w^{2}+s \lambda^{2} \varphi|\nabla w|^{2}\right) d x d t+\left\|\widetilde{g} e^{s \alpha}\right\|_{L^{2}(Q)}^{2}\right), \\
\forall s \geq s_{0} .
\end{gathered}
$$

Thus, from (7), (8) and (20), we have

$$
\begin{gathered}
\int_{Q}\left\{\frac{1}{s \varphi}\left(\frac{\partial w}{\partial t}\right)^{2}+\frac{1}{s \varphi} \sum_{i, j=1}^{n}\left(\frac{\partial^{2} w}{\partial x_{i} \partial x_{j}}\right)^{2}+s \lambda^{2} \varphi|\nabla w|^{2}+\lambda^{4} s^{3} \varphi^{3} w^{2}\right\} d x d t \\
\leq C\left(\int_{Q_{\omega}}\left(\lambda^{4} s^{3} \varphi^{3} w^{2}+s \lambda^{2} \varphi|\nabla w|^{2}\right) d x d t+\left\|\widetilde{g} e^{s \alpha}\right\|_{L^{2}(Q)}^{2}\right) \\
\forall s \geq s_{0} .
\end{gathered}
$$

Replacing $w$ by $e^{s \alpha} y$ in (21), we obtain

$$
\begin{gathered}
\int_{Q}\left(\frac{1}{s \varphi}\left(\frac{\partial y}{\partial t}\right)^{2}+\frac{1}{s \varphi} \sum_{i, j=1}^{n}\left(\frac{\partial^{2} y}{\partial x_{i} \partial x_{j}}\right)^{2}+s \lambda^{2} \varphi|\nabla y|^{2}+s^{3} \lambda^{4} \varphi^{3} y^{2}\right) e^{2 s \alpha} d x d t \\
\leq C_{1}(\lambda)\left(\int_{Q_{\omega}}\left(\lambda^{4} s^{3} \varphi^{3} y^{2}+s \lambda^{2} \varphi|\nabla y|^{2}\right) e^{2 s \alpha} d x d t+\left\|g e^{s \alpha}\right\|_{L^{2}(Q)}^{2}\right), \\
\forall s \geq s_{1} .
\end{gathered}
$$

Let us consider a function $\rho \in C_{0}^{\infty}(\omega), \rho(x) \equiv 1$ in $\omega_{0}$. We multiply equation (6) by $s \varphi \lambda^{2} y \rho e^{2 s \alpha}$ and take scalar products in $L^{2}(Q)$. Integrating by parts with respect to $t$ and $x$ and applying the Cauchy-Bunyakovskii inequality, we obtain

$$
\int_{(0, T) \times \omega_{0}} \lambda^{2} s \varphi|\nabla y|^{2} e^{2 s \alpha} d x d t \leq C\left(\int_{Q_{\omega}} \lambda^{4}(s \varphi)^{3} y^{2} e^{2 s \alpha} d x d t+\left\|\widetilde{g} e^{s \alpha}\right\|_{L^{2}(Q)}^{2}\right) .
$$


By virtue of (22) and (23), we have

(24)

$$
\begin{gathered}
\int_{Q}\left(\frac{1}{s \varphi}\left(\frac{\partial y}{\partial t}\right)^{2}+\frac{1}{s \varphi}\left(\sum_{i, j=1}^{n} \frac{\partial^{2} y}{\partial x_{i} \partial x_{j}}\right)^{2}+s \lambda^{2} \varphi|\nabla y|^{2}+s^{3} \lambda^{4} \varphi^{3} y^{2}\right) e^{2 s \alpha} d x d t \\
\leq C\left\{\int_{Q_{\omega}} \lambda^{4}(s \varphi)^{3} y^{2} e^{2 s \alpha} d x d t+\left\|g e^{s \alpha}\right\|_{L^{2}(Q)}^{2}\right\}, \quad \forall s \geq s_{0} .
\end{gathered}
$$

By (24), we finally obtain (2.11) with $d=0$.

\section{Appendix III}

Proof of Lemma 2.4. Since $a_{i j}, 1 \leq i, j \leq n$, are Lipschitz continuous on $\bar{Q}$, the unique existence of the solution in $L^{2}\left(0, T ; \stackrel{\circ}{W_{2}^{1}}(\Omega)\right) \cap C\left([0, T] ; L^{2}(\Omega)\right)$ is seen in the case of $b_{i}=0,1 \leq i \leq n$ and $c=0$, for example, by Ladyzenskaja, Solonnikov and Ural'ceva [35], Lions and Magenes [40, Chapter 3, Section 4.7], Pazy [43], Tanabe [51]. To prove the uniqueness of weak solutions to the problem (2.1)-(2.2) and a priori estimate (2.12), it suffices to prove that the problem

$$
L^{*} z=f,\left.\quad z\right|_{\Sigma}=0, \quad z(T, \cdot)=0
$$

has a solution $z \in L^{2}\left(0, T ; \stackrel{\circ}{W_{2}^{1}}(\Omega)\right) \cap C\left([0, T] ; L^{2}(\Omega)\right)$ for any $f \in L^{2}(0, T$; $\left.W_{2}^{-1}(\Omega)\right)$. To prove the solvability of problem (1), it is sufficient to prove the analogue of (2.12) for this problem.

Henceforth $C>0$ denotes a generic constant which is independent of functions to be estimated. Multiplication of (1) with $z$ and integration by parts in $x$ yield

$$
\begin{aligned}
-\frac{1}{2} \frac{d}{d t}\|z(t, \cdot)\|_{L^{2}(\Omega)}^{2} & +\int_{\Omega} \sum_{i, j=1}^{n} a_{i j} \frac{\partial z}{\partial x_{j}} \frac{\partial z}{\partial x_{i}} d x \\
& =\int_{\Omega} \sum_{i=1}^{n} b_{i} z \frac{\partial z}{\partial x_{i}} d x-\int_{\Omega} c z^{2} d x+\int_{\Omega} f z d x .
\end{aligned}
$$

By the uniform ellipticity, we see

(2) $-\frac{d}{d t}\|z(t, \cdot)\|_{L^{2}(\Omega)}^{2}+\|\nabla z(t, \cdot)\|_{L^{2}(\Omega)}^{2}$

$$
\leq \sum_{i=1}^{n}\left|\int_{\Omega} b_{i} z \frac{\partial z}{\partial x_{i}} d x\right|+\left|\int_{\Omega} c z^{2} d x\right|+\left|\int_{\Omega}\left(\frac{1}{\varepsilon} f\right)(\varepsilon z) d x\right| .
$$


Here $\varepsilon>0$ is a sufficiently small parameter which is fixed later, and we use $2|a b| \leq \varepsilon a^{2}+\frac{1}{\varepsilon} b^{2}$.

First we estimate $\sum_{i=1}^{n}\left|\int_{\Omega} b_{i} z \frac{\partial z}{\partial x_{i}} d x\right|$. We take $r>2 n$. By the Hölder inequality, we have

$$
\left|\int_{\Omega} b_{i} z \frac{\partial z}{\partial x_{i}} d x\right| \leq\left\|b_{i}(t, \cdot)\right\|_{L^{r}(\Omega)}\|z(t, \cdot)\|_{L^{\frac{2 r}{r-2}}(\Omega)}\left\|\frac{\partial z}{\partial x_{i}}(t, \cdot)\right\|_{L^{2}(\Omega)} .
$$

Since $r>2 n$, the Sobolev imbedding theorem implies $W_{2}^{\frac{1}{2}-\delta}(\Omega) \subset L^{\frac{2 r}{r-2}}(\Omega)$ for sufficiently small $\delta>0$. Hence, with small $\varepsilon>0$, we have

$$
\begin{aligned}
\left|\int_{\Omega} b_{i} z \frac{\partial z}{\partial x_{i}} d x\right| & \leq\|z(t, \cdot)\|_{W_{2}^{\frac{1}{2}-\delta}(\Omega)}\|z(t, \cdot)\|_{W_{2}^{1}(\Omega)} \\
& \leq \varepsilon\|z(t, \cdot)\|_{W_{2}^{1}(\Omega)}^{2}+\frac{C}{\varepsilon}\|z(t, \cdot)\|_{W_{2}^{\frac{1}{2}-\delta}(\Omega)}^{2}
\end{aligned}
$$

By the interpolation inequality, we see

$$
\|z(t, \cdot)\|_{W_{2}^{\frac{1}{2}-\delta}(\Omega)}^{2} \leq \delta\|z(t, \cdot)\|_{W_{2}^{1}(\Omega)}^{2}+C(\delta)\|z(t, \cdot)\|_{L^{2}(\Omega)}^{2}
$$

for small $\delta>0$. We choose sufficiently small $\varepsilon>0$ and $\delta>0$ such that $\frac{\delta}{\varepsilon}$ is also small, so that

$$
\sum_{i=1}^{n}\left|\int_{\Omega} b_{i} z \frac{\partial z}{\partial x_{i}} d x\right| \leq C \varepsilon\|z(t, \cdot)\|_{W_{2}^{1}(\Omega)}^{2}+C(\varepsilon)\|z(t, \cdot)\|_{L^{2}(\Omega)}^{2} .
$$

Now, by Lemma 2.2, we have

$$
\begin{aligned}
\left|\int_{\Omega} c z^{2} d x\right| & \leq\|c(t, \cdot)\|_{W_{r_{1}}^{-\mu}(\Omega)}\left\|z^{2}(t, \cdot)\right\|_{W_{r_{1}^{\prime}}^{\mu}(\Omega)} \\
& \leq C\|z(t, \cdot)\|_{W_{2}^{\frac{1}{2}-\delta}(\Omega)}\|z(t, \cdot)\|_{W_{2}^{1}(\Omega)} \\
& \leq C \varepsilon\|\nabla z(t, \cdot)\|_{L^{2}(\Omega)}^{2}+\frac{C}{\varepsilon}\|z(t, \cdot)\|_{W_{2}^{\frac{1}{2}-\delta}(\Omega)}^{2}
\end{aligned}
$$

with $0<\delta<\frac{1}{2}$. In view of interpolation inequality (3), taking $\varepsilon>0$ and $\delta>0$ so small that $\frac{\delta}{\varepsilon}$ is also small, we obtain

$$
\left|\int_{\Omega} c z^{2} d x\right| \leq \varepsilon\|\nabla z(t, \cdot)\|_{L^{2}(\Omega)}^{2}+C(\varepsilon)\|z(t, \cdot)\|_{L^{2}(\Omega)}^{2} .
$$

On the other hand, we have

(6) $\left|\int_{\Omega} f z d x\right|=\left|\int_{\Omega}(\varepsilon f)\left(\frac{1}{\varepsilon} z\right) d x\right| \leq C(\varepsilon)\|f(t, \cdot)\|_{W_{2}^{-1}(\Omega)}^{2}+\varepsilon\|\nabla z(t, \cdot)\|_{L^{2}(\Omega)}^{2}$. 
Applying (4)-(6) in (2), we have

$$
\begin{aligned}
-\frac{d}{d t} \int_{\Omega} z(t, \cdot)^{2} d x & +\|\nabla z(t, \cdot)\|_{L^{2}(\Omega)}^{2} \\
& \leq C \int_{\Omega} z(t, \cdot)^{2} d x+C\|f(t, \cdot)\|_{W_{2}^{-1}(\Omega)}^{2}, \quad t \geq 0 .
\end{aligned}
$$

In particular,

$$
-\frac{d}{d t} \int_{\Omega} z(t, \cdot)^{2} d x \leq C \int_{\Omega} z(t, \cdot)^{2} d x+C\|f(t, \cdot)\|_{W_{2}^{-1}(\Omega)}^{2}, \quad t \geq 0 .
$$

Hence by $z(T, \cdot)=0$, the Gronwall inequality implies

$$
\|z(t, \cdot)\|_{L^{2}(\Omega)}^{2} \leq C\|f\|_{L^{2}\left(0, T ; W_{2}^{-1}(\Omega)\right)}^{2}, \quad 0 \leq t \leq T .
$$

Integrating ( 7 ) in $t$ from 0 to $T$, we obtain

$$
\|z(0, \cdot)\|_{L^{2}(\Omega)}^{2}+\|\nabla z(t, \cdot)\|_{L^{2}(Q)}^{2} \leq C\|z\|_{L^{2}(Q)}^{2}+C\|f\|_{L^{2}\left(0, T ; W_{2}^{-1}(\Omega)\right)}^{2} .
$$

By (8) and (9), we have

$$
\|z\|_{L^{2}\left(0, T ; W_{2}^{1}(\Omega)\right)}^{2} \leq C\|f\|_{L^{2}\left(0, T ; W_{2}^{-1}(\Omega)\right)}^{2} .
$$

The proof of Lemma 2.4 is complete.

\section{Acknowledgements}

This paper has been partially established during the first named author's stay at The University of Tokyo in Japan and the Institute of Henry Poincaré in France. The authors wish to thank Professor J.-P. Puel for helpful discussions on the contents of this paper. The first named author was supported by KIAS under the grant number M97003. The second named author thanks the Korean Institute for Advanced Study in Seoul for arranging his stay which was useful for completing this paper. The authors thank the referee for valuable comments.

\section{References}

[1] Adams, R. A., Sobolev Spaces, Academic Press, New York, 1975.

[2] Alekseev, V. M., Tikhomirov, V. M. and Fomin, S. V., Optimal Control, Conlsultants Bureau, New York, 1987.

[3] Carleman, T., Sur un problème d'unicité pour les systèmes d'équations aux derivées partielles à deux variables independentes, Ark. Mat. Astr. Fys., 2B (1939), 1-9. 
[4] Chae, D., Imanuvilov, O. Yu. and Kim, S. M., Exact controllability for semilinear parabolic equations with Neumann boundary conditions, J. Dynam. Control Systems., 2 (1996), 449-483.

[5] Coron, J.-M., Contrôlabilité exacte frontière de l'équation d'Euler des fluides parfaits incompresibles bidimensionnels, C. R. Acad. Sci., Paris Sér I, 317 (1993), 271-276.

[6] - On the controllability of the 2-D incompressible Navier-Stokes equations with the Navier-slip boundary conditions, ESAIM: Control, Optimization and Calculus of Variations, 1 (1996), 35-75.

[7] - On the controllability of 2-D incompressible perfect fluids, J. Math. Pures Appl., 75 (1996), 155-188.

[8] Coron, J.-M. and Fursikov, A. V., Global exact controllability of the 2-D Navier-Stokes equations on manifold without boundary, Russian J. Math. Phys., 4 (1996), 1-20.

[9] Fabre, C., Uniqueness result for Stokes equations, ESAIM: Control, Optimization and Calculus of Variations, URL (http://www.emath.fr/cocv/) 1 (1996), 267-302.

[10] - Résultats d'unicité pour les équations de Stokes et applications au contrôle, $C$. R. Acad. Sci., Paris Sér I, 322 (1996), 1191-1196.

[11] Fabre, C. and Lebeau, G., Prolongement unique des solutions de l'équation de Stokes, Comm. Partial Differential Equations, 21 (1996), 573-596.

[12] Fabre, C., Puel, J.-P. and Zuazua, E., Contrôllabilité approchée de l'équation de la chaleur semi-linéaire, C. R. Acad. Sci. Paris Sér I, 315 (1992), 807-812.

[13] - Approximate controllability of the semilinear heat equation, Proc. Roy. Soc. Edinburgh Sect. A, 125 (1995), 31-61.

[14] Fernández, L. A. and Zuazua, E., Approximate controllability for the semilinear heat equation involving gradient terms, J. Optim. Theory Appl., 101 (1999), 307-328.

[15] Fernández-Cara E., Null controllability of the semilinear heat equation, ESAIM: Control, Optimization and Calculus of Variations, 2 (1997), 87-103.

[16] Fursikov, A. V. and Imanuvilov, O. Yu., On exact boundary zero-controllability of twodimensional Navier-Stokes equations, Acta Appl. Math., 37 (1994), 67-76.

[17] - On controllability of certain systems simulating a fluid flow, in Flow Control, IMA. Math. Appl., 68, edited by M. D. Gunzburger Springer-Verberg, New York, 1995, 148-184.

[18] - Local exact controllability of two dimensional Navier-Stokes system with control on the part of the boundary, Sbornik Mathematics, 187 (1996), 1355-1390.

[19] - Local exact controllability of the Navier-Stokes equations, C. R. Acad. Sci., Paris Sér I, 323 (1996), 275-280.

[20] Controllability of evolution equations, Lecture Notes Series, Seoul National University, Seoul, South Korea, 1996.

[21] - Local exact boundary controllability of the Boussinesq equation, SIAM J. Control Optim., 36 (1998), 391-421.

[22] Lin Guo, Y.-J. and Littman, W., Null boundary controllability for semilinear heat equations, Appl. Math. Optim., 32 (1995), 281-316.

[23] Hörmander, L., Linear Partial Differential Operators, Springer-Verlag, Berlin, 1963.

[24] - On the uniqueness of the Cauchy problem under partial analyticity assumptions, Geometrical Optics and Related Topics, edited by F. Colombini and N. Lerner, 32 (1997), 179-220.

[25] Imanuvilov, O. Yu., Boundary controllability of parabolic equations, Sbornik Mathematics, 186 (1995), 879-900.

[26] - On exact controllability for the Navier-Stokes equations, ESAIM: Control, Optimization and Calculus of Variations, 3 (1998), 97-131.

[27] Imanuvilov, O. Yu. and Yamamoto, M., Lipschitz stability in inverse parabolic problems by the Carleman estimate, Inverse Problems, 14 (1998), 1229-1245.

[28] Isakov, V., On the uniqueness of the solution of the Cauchy problem, Soviet. Math. Dokl., 22 (1980), 639-642.

[29] — Inverse Problems for Partial Differential Equations, Springer-Verlag, Berlin, 
1998.

[30] Jerison, D., Carleman inequalities for the dirac and Laplace operators and unique continuation, Adv. Math., 62 (1986), 118-134.

[31] Jerison, D. and Kenig, C. E., Unique continuation and absence of positive eigenvalues for Schrödinger operators, Ann. of Math., 121 (1985), 463-494.

[32] Kim, Y. M., Strong unique continuation of the Schrödinger operator, Bull. Korean Math. Soc., 31 (1994), 55-60.

[33] - Carleman inequalities for the dirac operator and strong unique continuation, Proc. Amer. Math. Soc., 123 (1995), 2103-2112.

[34] Kurata, K., On a backward estimate for solutions of parabolic differential equations and its applications to unique continuation, Adv. Stud. Pure Math., Spectral and Scattering Theory and Applications, 23 (1994), 247-257.

[35] Ladyzenskaja, O. A., Solonnikov, V. A. and Ural'ceva, N. N., Linear and Quasilinear Equations of Parabolic Type, American Mathematical Society, Providence, Rhode Island, 1968.

[36] Lavrent'ev, M. M., Romanov, V. G. and Shishat-skiı̌, S. P., Ill-posed Problems of Mathematical Physics and Analysis, American Mathematical Society, Providence, Rhode Island, 1986.

[37] Lin, F. H., A uniqueness theorem for parabolic equations, Comm. Pure Appl. Math., 43 (1990), 127-136.

[38] Lions, J. L., Optimal Control of Systems Governed by Partial Differential Equations, Springer-Verlag, Berlin, 1971.

[39] — Contrôle des Systèmes Distribués Singuliers, Gauthier-Villars, Paris, 1983.

[40] Lions, J. L. and Magenes, E., Non-Homogeneous Boundary Value Problems and Applications, Springer-Verlag, Berlin, 1972.

[41] Lu, G., Unique continuation with weak type lower order terms: the variable coefficient case, Publ. Mat., 39 (1995), 187-200.

[42] Mizohata, S., Le problème de Cauchy pour les systèmes hyperboliques et paraboliques, Mem. Coll. Sci. Univ. Kyoto, 32 (1959), 181-212.

[43] Pazy, A., Semigroups of Linear Operators and Applications to Partial Differential Equations, Springer-Verlag, Berlin, 1983.

[44] Poon, C.-C., Unique continuation for parabolic equations, Comm. Partial Differential Equations, 21 (1996), 521-539.

[45] Robbiano, L., Thèoréme d'unicité adapté au contôle des solutions des problèmes hyperboliques, Comm. Partial Differential Equations, 16 (1991), 789-800.

[46] Ruiz, A., Unique continuation for weak solutions of the wave equation plus a potential, J. Math. Pures Appl., 71 (1992), 455-467.

[47] Runst, T. and Sickel, W., Sobolev Spaces of Fractional Orders, Nemytskij Operators and Nonlinear Partial Differentional Equations, Walter de Gruyter, Berlin, 1996.

[48] Saut, J.-C. and Scheurer, B., Unique continuation for some evolution equations, $J$. Differential Equations, 66 (1987), 118-139.

[49] Sogge, C. D., Oscillatory integrals, Carleman inequalities and unique continuation for second order elliptic operators, J. Amer. Math. Soc., 2 (1989), 491-516.

[50] Unique continuation theorem for second order parabolic differential operators, Ark. Mat., 28 (1990), 159-182.

[51] Tanabe, H., Equations of Evolution, Pitman, London, 1979.

[52] Tataru, D., Carleman estimates and unique continuation for solutions to boundary value problems, J. Math. Pures Appl., 75 (1996), 367-408.

[53] - The $X_{\theta}^{s}$ spaces and unique continuation for solutions to the semilinear wave equation, Comm. Partial Differential Equations, 21 (1996), 841-887.

[54] Triebel, H., Interpolation Theory, Function Spaces, Differential Operators, second edition, Johann Ambrosius Barth Verlag, Heidelberg, 1995.

[55] Wolff, T. H., Recent work on sharp estimates in second-order elliptic unique continuation problems, J. Geom. Anal., 3 (1993), 621-650. 
[56] Zuazua, E., Approximate controllability of the semilinear heat equation: boundary control, International conference in honour of Prof. R. Glowinski, Computation Science for the 21st. Century, edited by M. O. Bristeau, (1997), 738-747. 